\title{
Clusters and superclusters in the Sloan Digital Sky Survey
}

\author{
J. Einasto ${ }^{1}$, G. Hütsi ${ }^{1}$, M. Einasto ${ }^{1}$, E. Saar ${ }^{1}$, D. L. Tucker ${ }^{2}$, V. Müller ${ }^{3}$, P. Heinämäki ${ }^{1,4}$, and S. S. Allam ${ }^{5,2}$ \\ 1 Tartu Observatory, 61602 Tõravere, Estonia \\ 2 Fermi National Accelerator Laboratory, MS 127, PO Box 500, Batavia, IL 60510, USA \\ 3 Astrophysical Institute Potsdam, An der Sternwarte 16, 14482 Potsdam, Germany \\ 4 Tuorla Observatory, Väisäläntie 20, Piikkiö, Finland \\ 5 National Research Institute for Astronomy \& Geophysics, Helwan Observatory, Cairo, Egypt
}

Received 9 December 2002 / Accepted 13 March 2003

\begin{abstract}
We apply the 2-dimensional high-resolution density field of galaxies of the Early Data Release of the Sloan Digital Sky Survey with a smoothing lengths $0.8 \mathrm{~h}^{-1} \mathrm{Mpc}$ to extract clusters and groups of galaxies, and a low-resolution field with smoothing lengths $10 \mathrm{~h}^{-1} \mathrm{Mpc}$ to extract superclusters of galaxies. We investigate properties of density field clusters and superclusters and compare properties of these clusters and superclusters with Abell clusters, and superclusters found on the basis of Abell clusters. We found that clusters in high-density environment have a luminosity a factor of 5-10 higher than in lowdensity environment. There exists a large anisotropy between the SDSS Northern and Southern sample in the properties of clusters and superclusters: most luminous clusters and superclusters in the Northern sample are a factor of 2 more luminous than the respective systems in the Southern sample.
\end{abstract}

Key words. cosmology: large-scale structure of the Universe - clusters of galaxies; cosmology: large-scale structure of the Universe - galaxies: clusters: general

\section{Introduction}

Clusters and groups of galaxies are the basic building blocks of the Universe on cosmological scales. The first catalogues of clusters of galaxies (Abell 1958; Zwicky et al. 1961-68) were constructed by visual inspection of the Palomar Observatory Sky Survey plates. More recent catalogues of clusters, as well as catalogues of groups of galaxies, have been derived using catalogues of galaxies (Huchra et al. 1983; Dalton et al. 1997). Moving up the hierarchy of large-scale structure, galaxy cluster catalogues themselves have been used to define still larger systems such as superclusters of galaxies (Oort 1983; Bahcall 1988; Einasto et al. 1994, 1997, 2001, hereafter E94, E97 and E01, Basilakos 2003).

The goal of the present series of papers is to map the Universe up to redshift $z=0.2$ and to find galaxy clusters and superclusters using the density field method. The application of the density field is not new. In the pioneering study by Davis \& Huchra (1982) the density field was used to calculate the gravitational field of the nearby Universe. Gott et al. (1986) used the density field to investigate topological properties of the Universe. Bertschinger et al. (1990) calculated the potential, velocity, and density fields from redshift-distance data. Saunders et al. (1991) applied the density field to map the Universe, to find superclusters and voids, and calculated

Send offprint requests to: J. Einasto, e-mail: einasto@aai.ee moments of the density field. Marinoni et al. (1999) reconstructed the real space local density field. In these studies nearby optical or infrared galaxy samples were used. More recently, Hoyle et al. (2002) used smoothed 2-dimensional density fields from volume limited subsamples of Sloan Digital Sky Survey Early Data Release (SDSS EDR) galaxies to discuss the 2-dimensional geometry of the large-scale matter distribution in comparison with $\Lambda \mathrm{CDM}$ simulations, and Sheth et al. (2002) advertised a new method of evaluating isodensity contours of smoothed 3-dimensional density fields from simulations for characterising topological properties of the supercluster-void network.

In the present paper we calculate the density field of the Sloan Digital Sky Survey Early Data Release by Stoughton et al. (2002) to find clusters and superclusters of galaxies, and to investigate their properties. Clusters of galaxies from the SDSS were extracted previously by Goto et al. (2002) using the cut and enhance method; Kim et al. (2002) compared various cluster detection algorithms based on SDSS data. In this paper we define clusters as enhancements of the density field and use various smoothing lengths to separate systems of galaxies of different size and luminosity. We use a high-resolution density field to find clusters and groups of galaxies. For simplicity, we use the term "DF-clusters" for both groups and clusters found in the high-resolution density field of galaxies. Similarly, we use a low-resolution density field to construct a catalogue 
Table 1. Data on SDSS EDR galaxies, clusters and superclusters.

\begin{tabular}{cccccccccccc}
\hline \hline Sample & DEC & RA & $\Delta$ RA & $\alpha_{1}$ & $M_{1}^{*}$ & $\alpha_{2}$ & $M_{2}^{*}$ & $N_{\text {gal }}$ & $N_{\text {cl }}$ & $N_{\text {ACO }}$ & $N_{\text {scl }}$ \\
\hline SDSS.N & $0^{\circ}$ & $190.25^{\circ}$ & $90.5^{\circ}$ & -1.06 & -21.55 & -1.22 & -20.80 & 15209 & 2868 & 22 & 24 \\
SDSS.S & $0^{\circ}$ & $23.25^{\circ}$ & $65.5^{\circ}$ & -1.06 & -21.40 & -1.10 & -20.71 & 11882 & 2287 & 16 & 16 \\
\hline
\end{tabular}

of superclusters of galaxies, and denote them as "DFsuperclusters". DF-clusters and superclusters are defined as enhancements of the density field, DF-clusters in a fixed volume $\left( \pm 2.5 h^{-1} \mathrm{Mpc}\right.$ from the centre), and DF-superclusters as highdensity regions surrounded by a fixed isodensity contour. Here and elsewhere in this paper, the Hubble constant is expressed as usual in units of $H_{0}=h 100 \mathrm{~km} \mathrm{~s}^{-1} \mathrm{Mpc}^{-1}$. In determining DF-clusters and superclusters we take into account known selection effects. We shall investigate some properties of DFclusters and superclusters, and study these clusters and superclusters as tracers of the structure of the local Universe. The use of the density field allows us to detect many systems of galaxies, especially poor systems, previously not catalogued, and in this way get a better picture of the distribution and properties of systems of galaxies. But this advantage has its price - some DF-clusters may be dynamically unstable, and mark just the present situation in the evolution of the structure.

This study is of exploratory character to find the potential of SDSS data to analyse the structure of the Universe both on small and large scales. In this stage we use the fact that SDSS EDR covers only relatively thin slices (at the outer edge of the survey, $z=0.2$, the slices have a thickness only $24 h^{-1} \mathrm{Mpc}$ ); thus we calculate the density field in 2 dimensions only. As more data will be made available we plan to use a full 3dimensional data set to detect clusters and superclusters of galaxies. In this exploratory stage of the study we will make no attempt to convert distances of galaxies and systems of galaxies from redshift space to real space. This correction applies only to positions of galaxies and clusters, not to their luminosity. Due to smoothing of the density field, small-scale corrections (e.g., the "finger-of-god" effect) are essentially flattened out.

Although there are large-scale corrections due to the apparent contraction of superclusters in redshift space (Kaiser 1984), the actual positional shifts of supercluster centres are very small; furthermore, they do not alter cluster and galaxy positions in tangential direction. The only large-scale redshift distortion is the radial contraction of superclusters, not the number and luminosity of clusters within superclusters. Since supercluster shapes are not the main target of this present study, we may ignore this effect for now.

In calculation of weights of galaxies we apply parameters of the luminosity function of galaxies as found by Hütsi et al. (2003, hereafter H03). The analysis of the density field of the Las Campanas Redshift Survey (LCRS) shall be published by Einasto et al. (2003b, hereafter Paper II). The comparison of observational results with numerical simulations shall be done in a separate paper (in preparation). There we shall use mock galaxy catalogues and results of $\mathrm{N}$-body simulations of the evolution of the structure to check various aspects of the density field method, and try to find theoretical explanation of observational results of this paper and Paper II. This series of papers is carried out in the framework of preparations for the analysis of results of the Planck mission to observe the Cosmic Microwave Background radiation.

The present paper is organised as follows. In Sect. 2 we give an overview of the observational data. In Sect. 3 we find DFclusters and investigate their properties. Similarly, in Sect. 4 we compose a catalogue of DF-superclusters, identify them with conventional superclusters, and study their properties. In Sect. 5 we continue the study of DF-clusters and superclusters, derive the luminosity function of DF-clusters, and analyse these systems as tracers of the large-scale structure of the universe. Section 6 brings our conclusions. The three-dimensional distribution of DF-clusters and superclusters in comparison with Abell clusters and superclusters is shown on the web-site of Tartu Observatory (http: //www . aai . ee).

\section{Observational data}

\subsection{SDSS Early Data Release}

The SDSS Early Data Release consists of two slices of about 2.5 degrees thickness and 65-90 degrees width, centred on celestial equator, one in the Northern and the other in the Southern Galactic hemisphere (Stoughton et al. 2002). This data set contains over 30000 galaxies with measured redshifts. We obtained from the SDSS Catalogue Archive Server angular positions, Petrosian magnitudes, and other available data for all EDR galaxies. From this general sample we extracted the Northern and Southern slice samples using following criteria: redshift interval $1000 \leq c z \leq 60000 \mathrm{~km} \mathrm{~s}^{-1}$, Petrosian $r^{*}$ magnitude interval $13.0 \leq r^{*} \leq 17.7$, right ascension and declination interval $140^{\circ} \leq \mathrm{RA} \leq 240.0^{\circ}$ and $-1.2^{\circ} \leq \mathrm{DEC} \leq 1.2^{\circ}$ for the Northern slice, and $340^{\circ} \leq \mathrm{RA} \leq 60.0^{\circ}$ and $-1.25^{\circ} \leq$ $\mathrm{DEC} \leq 1.25^{\circ}$ for the Southern slice. The number of galaxies extracted and the mean RA and DEC are given in Table 1. Observed redshifts were first corrected for the motion relative to the CMB dipole (Lineweaver et al. 1996). Co-moving distances of galaxies were calculated using a cosmological model with density parameters: matter density $\Omega_{m}=0.3$, dark energy density $\Omega_{\Lambda}=0.7$, total density $\Omega_{0}=\Omega_{m}+\Omega_{\Lambda}=1.0$, all in units of the critical cosmological density (see Peacock 1999). With these parameters the limiting redshift $z_{\lim }=0.2$ corresponds to co-moving distance $r_{\text {lim }}=571 \mathrm{~h}^{-1} \mathrm{Mpc}$. In calculating absolute magnitudes we used K-corrections and correction for absorption in the Milky Way (for details see H03). 


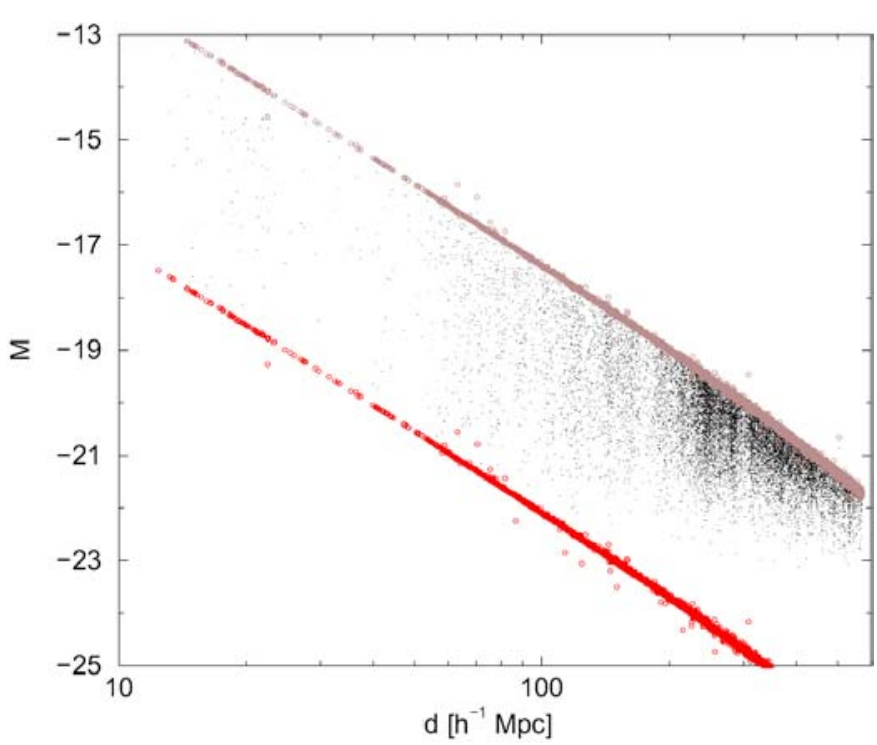

Fig. 1. Absolute magnitudes of galaxies (black dots), and magnitudes of the luminosity window, $M_{1}$ and $M_{2}$ (grey dots aligned in almost straight lines), for the Northern slice.

\subsection{Abell clusters and superclusters}

We shall use the sample of rich clusters of galaxies by Abell (1958) and Abell et al. (1989) (hereafter Abell clusters), compiled by Andernach \& Tago (1998), with redshifts up to $z=$ 0.13 . The sample contains 1665 clusters, 1071 of which have measured redshifts for at least two galaxies. This sample was described in detail by E01, where an updated supercluster catalogue of Abell clusters was presented. Superclusters were identified using a friend-of-friends algorithm with a neighbourhood radius of $24 h^{-1} \mathrm{Mpc}$. In the density field map (Fig. 4) we have used a new compilation of redshifts of Abell clusters by Andernach and Tago.

\section{Density field clusters}

We shall apply the density field to find density enhancements - DF-clusters. The procedure consists of several steps: (1) calculation of the distance, absolute magnitude, and weight factor for each galaxy of the sample; (2) calculation of rectangular coordinates of galaxies and rotation of coordinate axes in order to minimise projection effects; (3) smoothing of the density field using an appropriate kernel and smoothing length, and (4) finding clusters (density enhancements) in the field. Our method of cluster finding assumes that clusters lie completely within the slice, and that there are no other clusters with identical $x, y$-coordinates but shifted vertically. These assumptions are fulfilled in most cases. The extraction of clusters in the SDSS using full 3-dimensional density field information is planned in the future.

In calculating the density field we make two assumptions. First, we regard every galaxy as a visible member of a density enhancement (group or cluster) within the visible range of absolute magnitudes, $M_{1}$ and $M_{2}$, corresponding at the distance of the galaxy to the observational window of apparent magnitudes. This assumption is based on observations of nearby galaxies, which indicate that there are really almost no isolated galaxies except perhaps very low-luminosity and diffuse galaxies not represented in surveys like the SDSS. Most galaxies belong to poor groups like our own Galaxy, where one bright galaxy is surrounded by a number of faint satellites. Using these assumptions we actually find halos, either halos of single giant galaxies with their companions, or halos of group or clusters. Further, we assume that the luminosity function derived for a representative volume can be applied also for individual groups and galaxies. As we shall see later, this last assumption is not correct, and we must use various galaxy luminosity functions in order to calculate density fields suitable for finding DF-clusters and DF-superclusters.

The expected total number of galaxies per one visible galaxy is proportional to the inverse of the selection function,

$N_{\text {tot }}=W_{N}$

where

$W_{N}=\frac{\int_{0}^{\infty} \phi(L) \mathrm{d} L}{\int_{L_{1}}^{L_{2}} \phi(L) \mathrm{d} L}$

is the number-density weight. In the last equation $L_{i}=$ $L_{\odot} 10^{0.4 \times\left(M_{\odot}-M_{i}\right)}$ are luminosities of the observational window corresponding to absolute magnitudes of the window $M_{i}$ (see Fig. 1), and $M_{\odot}$ is the absolute magnitude of the Sun. The number-density weigh $W_{N}$ is shown in Fig. 2.

We are interested to find total luminosities of DF-clusters and superclusters, thus we shall use in the following analysis the luminosity density rather than the number density. The estimated total luminosity per one visible galaxy is

$L_{\mathrm{tot}}=L_{\mathrm{obs}} W_{L}$

where $L_{\mathrm{obs}}=L_{\odot} 10^{0.4 \times\left(M_{\odot}-M\right)}$ is the luminosity of the visible galaxy of absolute magnitude $M$, and

$W_{L}=\frac{\int_{0}^{\infty} L \phi(L) \mathrm{d} L}{\int_{L_{1}}^{L_{2}} L \phi(L) \mathrm{d} L}$

is the luminous-density weight (the ratio of the expected total luminosity to the expected luminosity in the visibility window). In calculation of weights we assume that galaxy luminosities are distributed according to the Schechter (1976) luminosity function:

$\phi(L) \mathrm{d} L \propto\left(L / L^{*}\right)^{\alpha} \exp \left(-L / L^{*}\right) \mathrm{d}\left(L / L^{*}\right)$

where $\alpha$ and $L^{*}$ are parameters. We used values of parameters as found by $\mathrm{H} 03$ and given in Table 1 . This procedure was also used by Tucker et al. (2000) in the calculation of total luminosities of groups of galaxies. A different procedure was applied by Moore et al. (1993) - adding the expected luminosity of faint galaxies outside the observational window to the luminosity of the observed galaxy. We assume that density and luminosity distributions are independent, which leads to a multiplicative correction (3). 

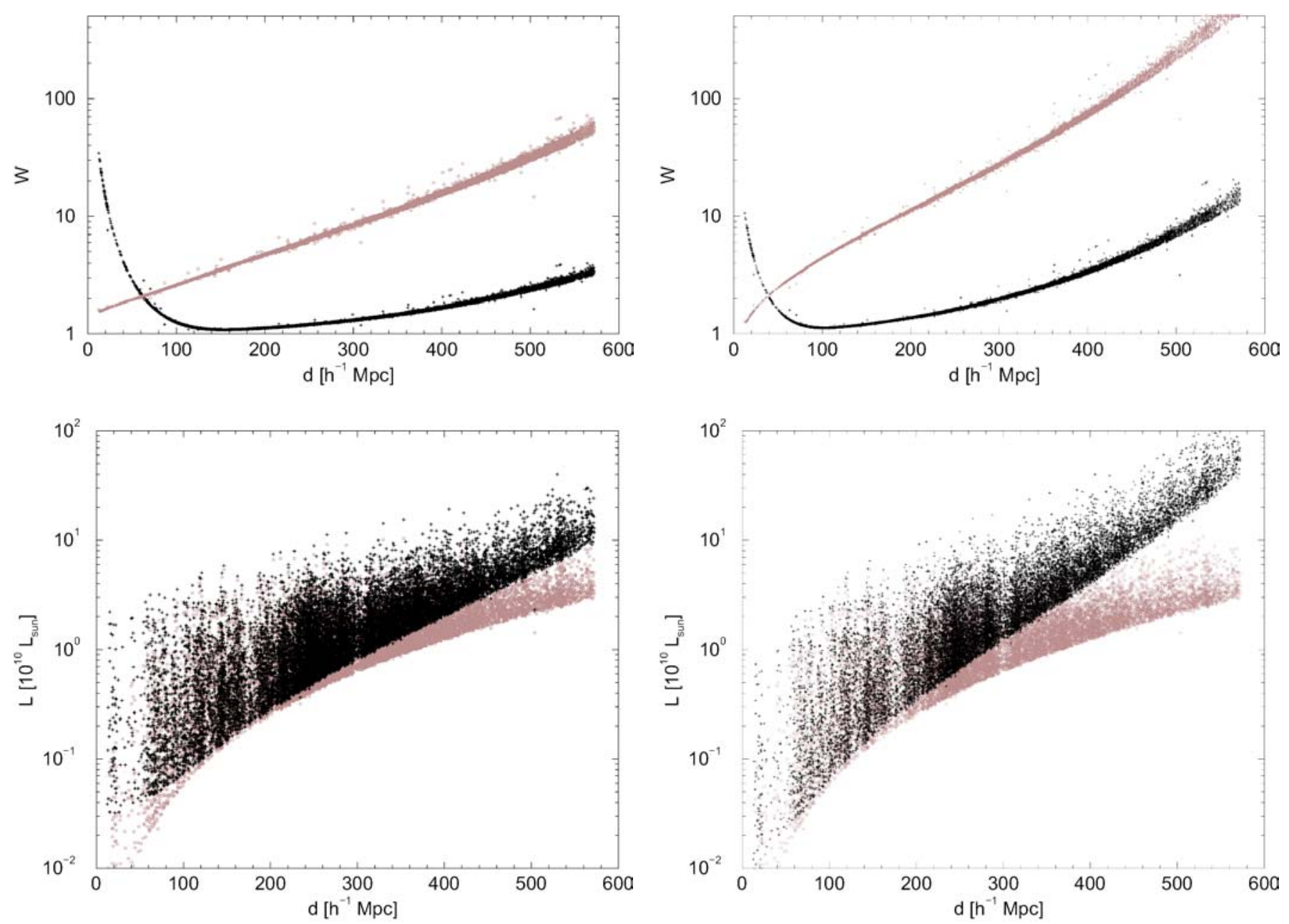

Fig. 2. Upper panels show weights as a function of distance. Grey symbols indicate number-density weights, black symbols luminous-density weights. Lower panels plot luminosities of galaxies as a function of distance. Grey circles mark luminosities of observed galaxies, black symbols total luminosities, corrected for unobservable part of the luminosity range, Eq. (3). Left panels are for luminosity function parameters of set 1 , and right panels for set 2 , which yield better DF-clusters and DF-superclusters, respectively.

As an example, we plot in Fig. 1 for the Northern slice the absolute magnitudes of the window, $M_{1}$ and $M_{2}$, as well as observed absolute magnitudes of galaxies, $M_{\text {obs }}$. In upper panels of Fig. 2 we show luminous-density weights $W_{\mathrm{L}}$ for both sets of the luminosity function. For comparison we show also numberdensity weights $W_{\mathrm{N}}$. Lower panels of Fig. 2 show luminosities of galaxies $L_{\mathrm{obs}}$, and expected total luminosities $L_{\mathrm{tot}}$ calculated with Eq. (3). Luminosities are expressed in units of $10^{10}$ Solar luminosities. We see that the number-density weight $W_{\mathrm{N}}$ rises monotonically with increasing distance from the observer, whereas the luminous-density weight $W_{\mathrm{L}}$ rises also toward very small distances. This is due to the influence of bright galaxies outside the observational window, which are not numerous (see the weight $W_{\mathrm{N}}$ ) but are very luminous. On larger distances the weight $W_{\mathrm{L}}$ rises again due to the influence of faint galaxies outside the observational window. In calculation of the relative density field only densities in units of the mean density matter, and in this respect the mean distance dependence of $L_{\text {tot }}$ is not very different from the distance dependence of the numberdensity $N_{\text {tot }}$.
To find the luminous-density field we calculated for all galaxies rectangular equatorial coordinates as follows:

$$
\begin{aligned}
& x=r\left(z_{\mathrm{c}}\right) \cos \mathrm{DEC} \cos \mathrm{RA}, \\
& y=r\left(z_{\mathrm{c}}\right) \cos \mathrm{DEC} \sin \mathrm{RA}, \\
& z=r\left(z_{\mathrm{c}}\right) \sin \mathrm{DEC},
\end{aligned}
$$

here RA and DEC are equatorial coordinates of the galaxy, $r\left(z_{\mathrm{c}}\right)$ is its comoving distance, and $z_{\mathrm{c}}$ is the redshift, corrected for the motion relative to $\mathrm{CMB}$ dipole. Next we rotated this coordinate system around the $z$-axis to obtain a situation where the new $y$-axis were oriented toward the average right ascension of each slice (see Table 1) and finally we rotated this new system around its $x$-axis so as to force the average $z$-coordinate to be zero in order to minimise projection effects (in case of SDSS EDR the last rotation angle is zero since data are located along the celestial equator, but for LCRS this angle is rather large, see Paper II).

To smooth the density field two approaches are often used. The first one uses top-hat smoothing as customary in $N$-body calculations. In this case the density in grid corners is found 
by linear interpolation where the "mass" of the particle is divided between grid corners according to the distance from the respective corner. Variable smoothing length can be obtained by changing the size of the grid cell. A better and smoother density field can be obtained when we use Gaussian smoothing. In this case the weight of the galaxy is distributed between neighbouring cells using the Gaussian smoothing

$W_{j g}=\left(2 \pi \sigma_{\mathrm{sm}}^{2}\right)^{-\frac{1}{2}} \exp \left(-\frac{\left|\boldsymbol{x}_{j}-\boldsymbol{x}_{\mathrm{g}}\right|^{2}}{2 \sigma_{\mathrm{sm}}^{2}}\right)$,

here $\boldsymbol{x}_{j}$ and $\boldsymbol{x}_{\mathrm{g}}$ are positions of the galaxy and the grid cell, respectively, and $\sigma_{\mathrm{sm}}$ is the smoothing length. To calculate the density fields, we formed a grid of cell size $1 \mathrm{~h}^{-1} \mathrm{Mpc}$, and used only $x, y$-coordinates. Ignoring the $z$-coordinate is equivalent to integrating over this coordinate, i.e. we find actually the projected density of the wedge. Since the thickness of the wedge increases with the distance from the observer, the projected density increases too. To compensate this effect and to find the spatial density we divide the projected density by the thickness of the wedge at the particular distance. This is not done for the high-resolution field. Here the probability of vertical projection of clusters is small (see Sect. 5.7 for further analysis), and by dividing the density by the thickness we distort luminosities of DF-clusters.

To find the proper value of the smoothing length we performed a test study. With increasing smoothing length we can find systems of galaxies of increasing scale from clusters and groups and galaxy and cluster filaments to superclusters and their aggregates. Figure 3 shows the density field of the Northern slice calculated with dispersions $0.8 \ldots 16 h^{-1} \mathrm{Mpc}$. To find DF-clusters with properties close to those of conventional clusters and groups a high-resolution density field is to be used with smoothing scale comparable to the characteristic scale of clusters and groups. The harmonic mean radius of groups and clusters in the Las Campanas Redshift Survey lies in the range $0.45-0.65 h^{-1} \mathrm{Mpc}$ (Tucker et al. 2000; Einasto et al. 2003a, hereafter E03). Abell, APM, and X-ray clusters which have been matched to Las Campanas clusters have harmonic mean radii in the same range. We have used a larger smoothing length, $0.8 \mathrm{~h}^{-1} \mathrm{Mpc}$, to identify DF-clusters; this smoothing length is equal to the upper quartile of harmonic radii of groups and clusters (E03). With this smoothing length and Gaussian density profile we get density distributions which match satisfactorily surface density profiles of clusters of galaxies (Carlberg et al. 1997). Thus we get a reasonable size of groups even in the case when groups are represented by only a single bright galaxy (we repeat that we consider every galaxy as a representative of a group which includes galaxies outside the observational magnitude window). Most massive clusters in our sample are represented by a number of galaxies with a spread in spatial positions. Thus a modest Gaussian widening does not considerably distort the actual profile of the cluster. After the Gaussian smoothing we get density maps shown in Figs. 3-5.

The final step is the identification of the DF-clusters. To identify DF-clusters of galaxies, every cell of the field was checked to see whether the density of the cell exceeded the density of all neighbouring cells. If the density of the cell was higher than that of all its neighbours, then the cell was considered as the centre of a DF-cluster and its $x, y$-coordinates, density value, and luminosity were measured. Inspection of the density field has shown that practically all compact systems of galaxies (groups and clusters) contain their luminosity within a box of size $-2 \leq \Delta x \leq 2$, and $-2 \leq \Delta y \leq 2$ in cell size units $\left(1 h^{-1} \mathrm{Mpc}\right)$; smoothing with scale $0.8 h^{-1} \mathrm{Mpc}$ spreads the luminous-density just between these cells. The total luminosity of the DF-cluster was derived by adding luminosity densities of the 24 surrounding cells to the luminosity density of the central cell. All densities and luminosities were calculated in Solar luminosity units. We also calculated the density in units of the mean density, this relative density was used in Figs. 3-5 for plotting. A cluster was added to the list of DF-clusters if its luminosity $\mathcal{L}$ exceeds a certain threshold value; we used the threshold $\mathcal{L}_{0}=0.4 \times 10^{10} L_{\odot}$. This threshold exceeds the luminosity of poorest groups by a factor of about 2, the number of groups below this limit is very small, see Fig. 6 . All used cells were marked and in the search for new clusters cells already counted were not included. Figure 4 shows that there are only a few galaxies in the nearby region, $d<100 h^{-1} \mathrm{Mpc}$. Moreover, near the outer boundary of the sample, $d>550 h^{-1} \mathrm{Mpc}$, samples are already diluted. Therefore, we shall confine our DF-cluster catalogue to the distance interval $100 \leq d \leq 550 \mathrm{~h}^{-1} \mathrm{Mpc}$. The total number of DF-clusters found is given in Table 1.

Now we look at some properties of DF-clusters. Figure 6 shows luminosities of DF-clusters as a function of distance from the observer, $d$. The lowest luminosity clusters are seen only at a distance $d \leq 150 h^{-1} \mathrm{Mpc}$. There exists a well-defined lower limit of cluster luminosities at larger distances, the limit being linear in the $\log L-d$ plot. Such behaviour is expected as at large distances an increasing fraction of clusters does not contain any galaxies bright enough to fall into the observational window of absolute magnitudes, $M_{1} \ldots M_{2}$. The cluster lower luminosity limit is somewhat lower for the Southern slice. The reason for this difference is not yet clear. Nonetheless, the absence of low-luminosity clusters at large distances can be taken into account in calculation of the cluster luminosity function (see Sect. 5 below).

According to general cosmological principles, the mean density of luminous matter (smoothed over superclusters and voids) should be the same everywhere. Some weak dependence on distance may be due to evolutionary effects: luminosities of non-interacting galaxies decrease as stars age; but luminosities of merging galaxies (central galaxies of clusters) increase with age. Both effects are rather small within the redshift range $z \leq 0.2$ used in this work (Shepherd et al. 2000). If we ignore these effects we may expect that the luminous density should not depend on the distance from the observer, in contrast to the number of galaxies which is strongly affected by selection effects (at large distances we do not see low-luminosity galaxies). This difference between the observed and total luminosity is well seen in Fig. 2: with increasing distance the weight $W_{L}$ (the ratio of the total-to-observed luminosity) increases by a factor of 3 (parameter set 1) or even 15 (parameter set 2). 

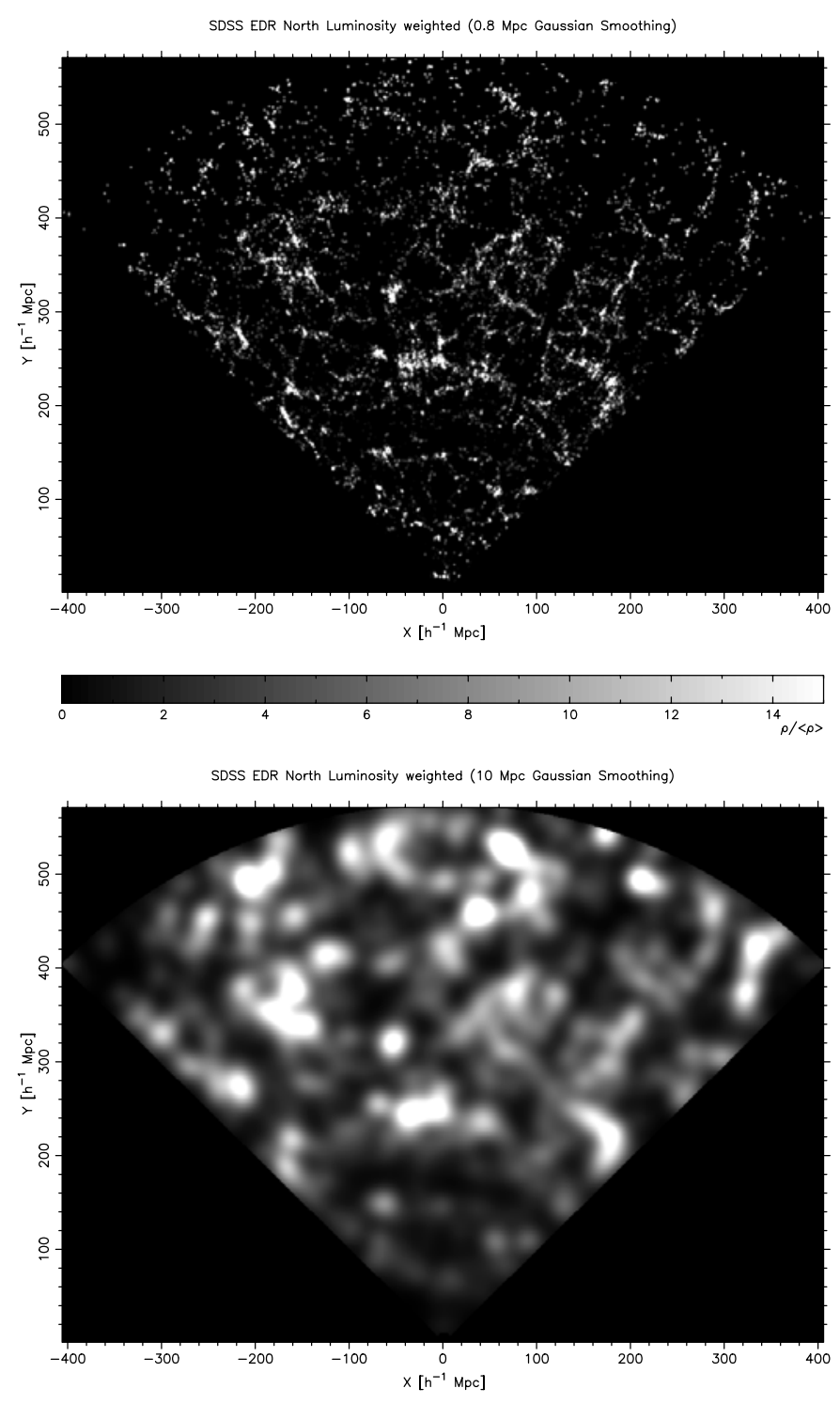

0.5
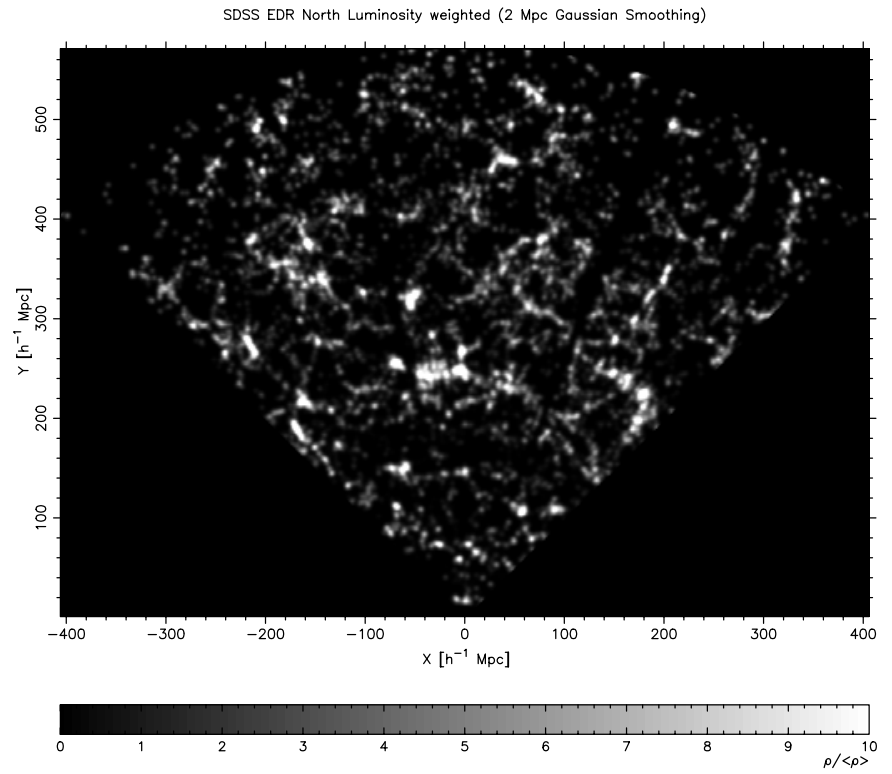

SDSS EDR North Luminosity weighted (16 Mpc Goussion Smoothing)

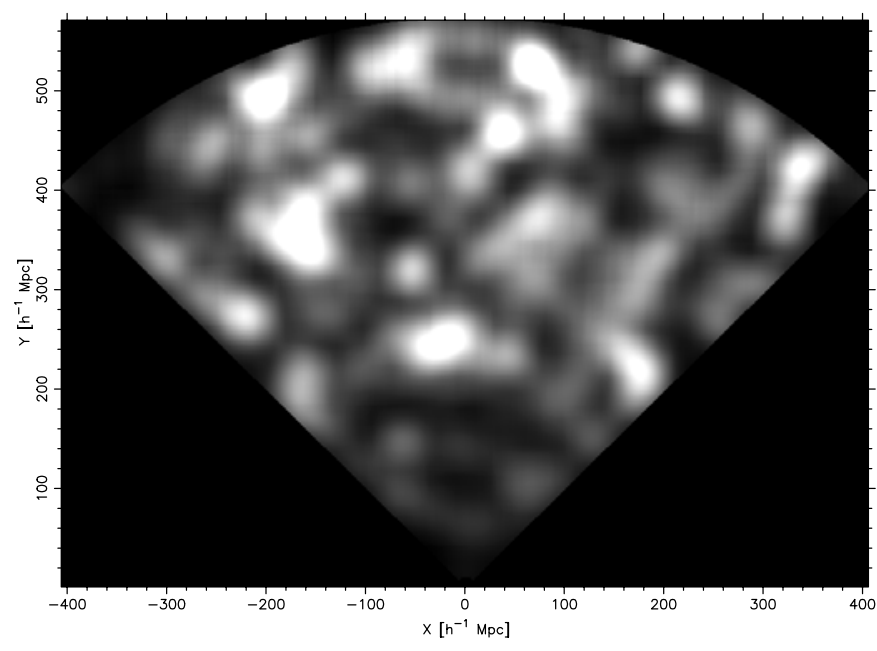

0.5

Fig. 3. Density field of the SDSS EDR Northern slice, smoothed with $\sigma=0.8 h^{-1} \mathrm{Mpc}, 2 h^{-1} \mathrm{Mpc}, 10 h^{-1} \mathrm{Mpc}$, and $16 h^{-1} \mathrm{Mpc}$ dispersion. Upper panels were calculated for parameter set 1 (better reproduction of clusters), lower panels for set 2 (better reproduction of superclusters). In all cases the density field was reduced to a sheet of constant thickness.

We can use the mean total luminous density as a test of our weighting procedure. The mean luminous density in a shell between distances $d_{i}$ and $d_{i-1}$ was found as follows:

$D_{L}\left(d_{i}\right)=L_{i} /\left(\frac{1}{3} \omega\left(d_{i}^{3}-d_{i-1}^{3}\right)\right)$

where $L_{i}$ is the sum of total luminosities of all DF-clusters in the shell, and $\omega$ is the angular size of the slice in sterradians. In Fig. 7 we show the mean luminous density in spherical shells of radial thickness $5 h^{-1} \mathrm{Mpc}$ for $\mathrm{N}$ and $\mathrm{S}$ slices of the SDSS EDR. We see strong fluctuations of the mean luminous density due to superclusters and voids. The nearby volume is small, so density fluctuations are large at distances $d \leq 220 h^{-1} \mathrm{Mpc}$; here we also see a low-density region in the Northern slice (see also Figs. 3-5). At large distances, the overall mean density for parameter set 2 is almost constant. However, in this case the luminosity of clusters rises considerably with distance, and all very luminous clusters are concentrated near the outer border of samples (see Figs. 4 and 6).

In determination of the parameter set 2 the number of faint unobservable galaxies in calculation of the luminosity function was estimated using Schechter parameters for the whole sample. As we have seen above, faint clusters are not visible at large distances. Thus, in order to estimate correctly the total number of faint galaxies, the luminous mass of invisible clusters has been added to visible clusters, and the luminosity of visible clusters has been overestimated. To get correct luminosities of clusters we have used the parameter set 1 which yields more or less uniform level of high-mass clusters at various 
SDSS EDR North and South (0.8 Mpc Gaussian Smoothing)
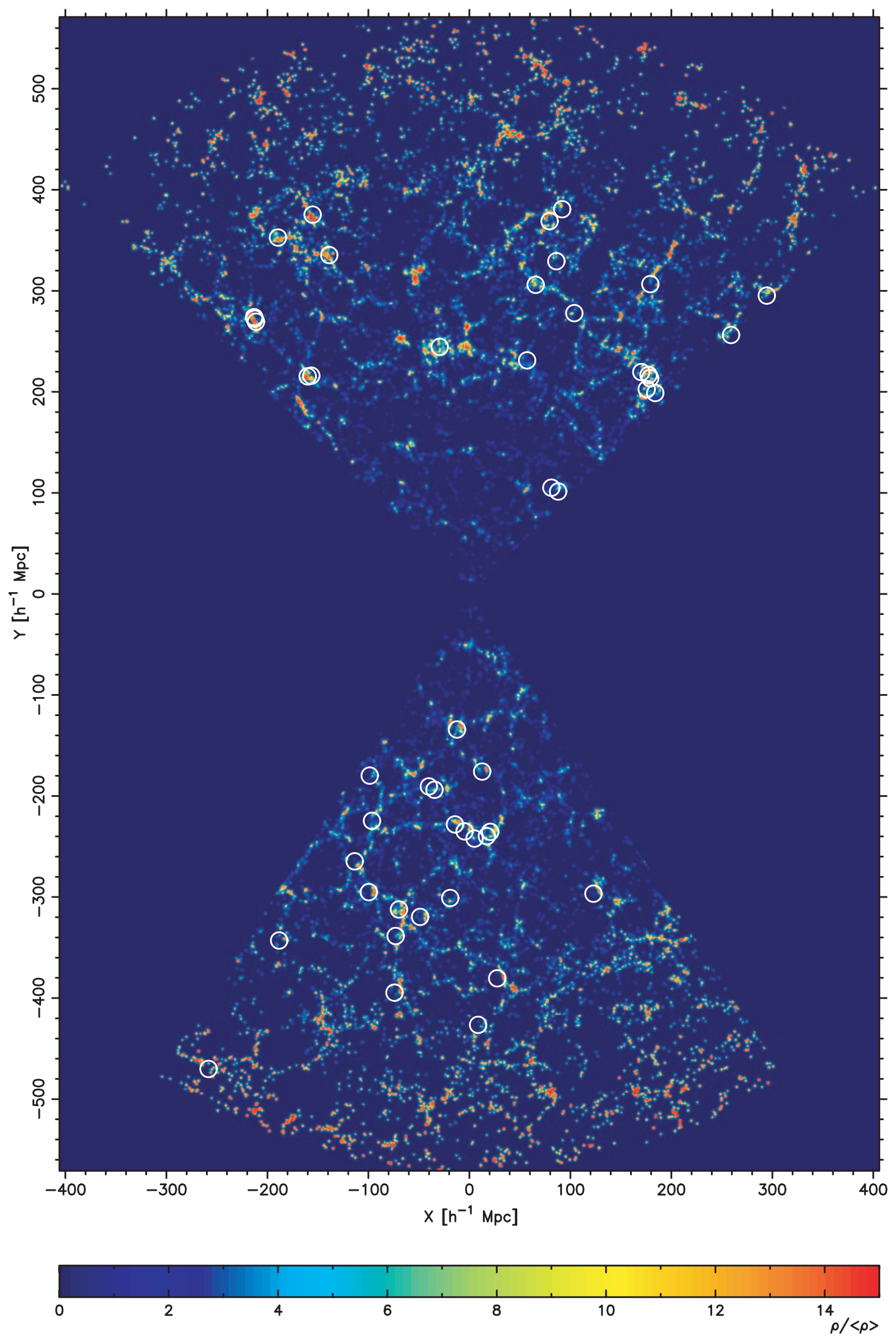

Fig. 4. Density field of the SDSS EDR slices, smoothed with $\sigma=0.8 \mathrm{~h}^{-1} \mathrm{Mpc}$ dispersion. Open circles note positions of Abell clusters located within boundaries of slices. In some cases an Abell cluster consists of several subclusters, in these cases only rich subclusters are marked. 
SDSS EDR North and South (10 Mpc Gaussian Smoothing)

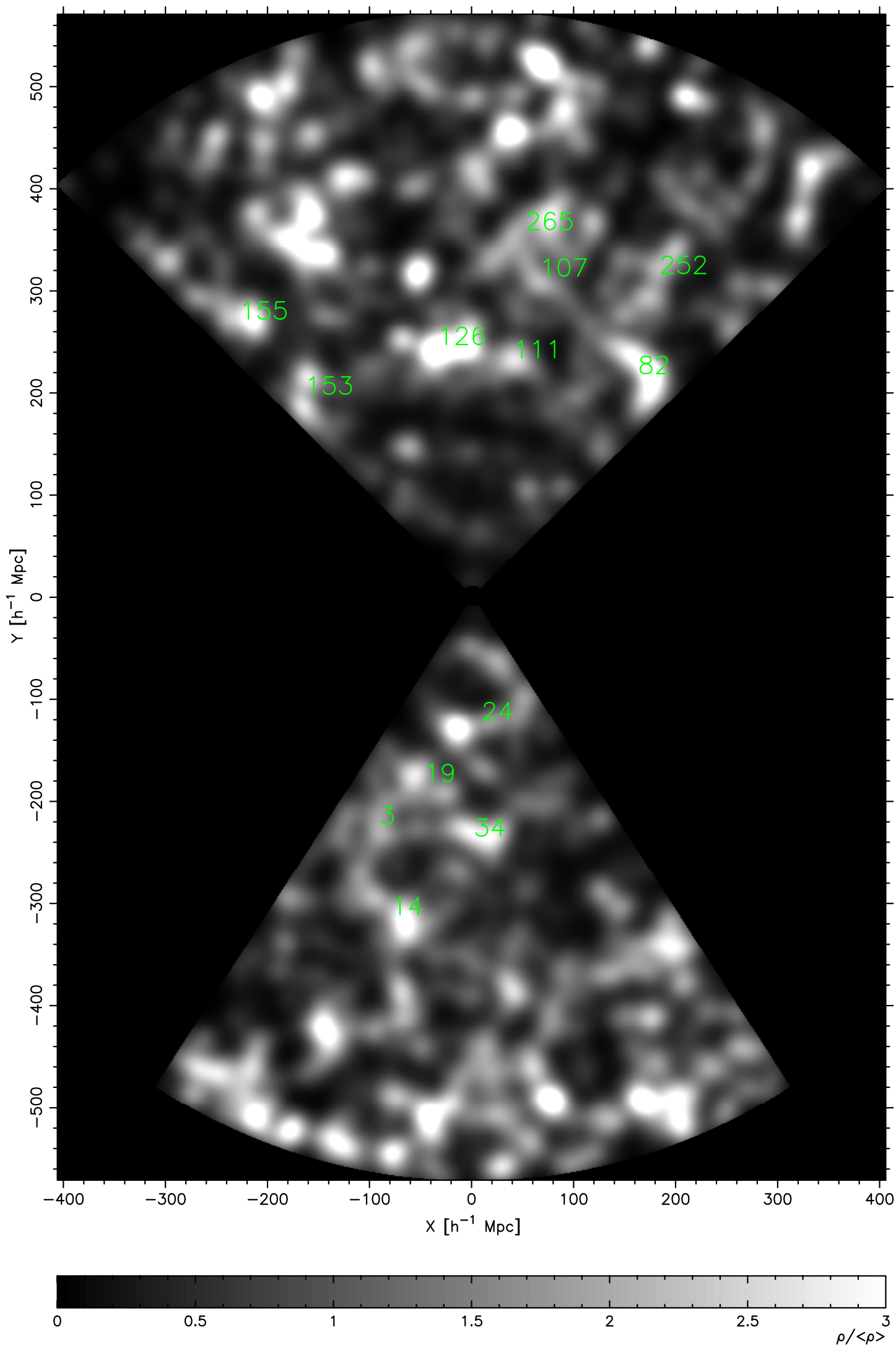

Fig. 5. Density field of the SDSS EDR slices, smoothed with $\sigma=10 h^{-1} \mathrm{Mpc}$ dispersion. Numbers show Abell superclusters according to E01. 
N1

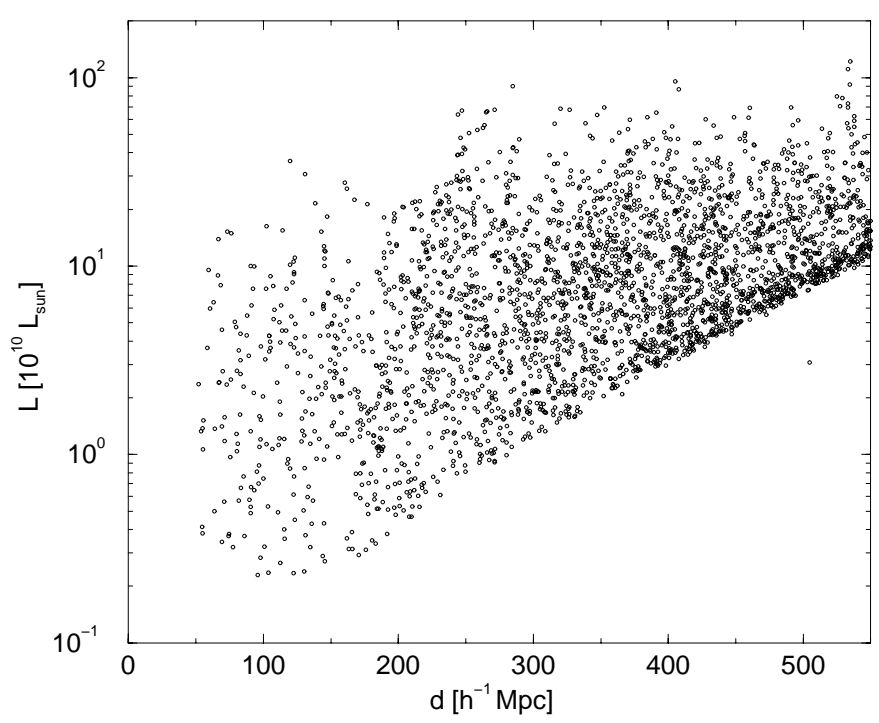

S1

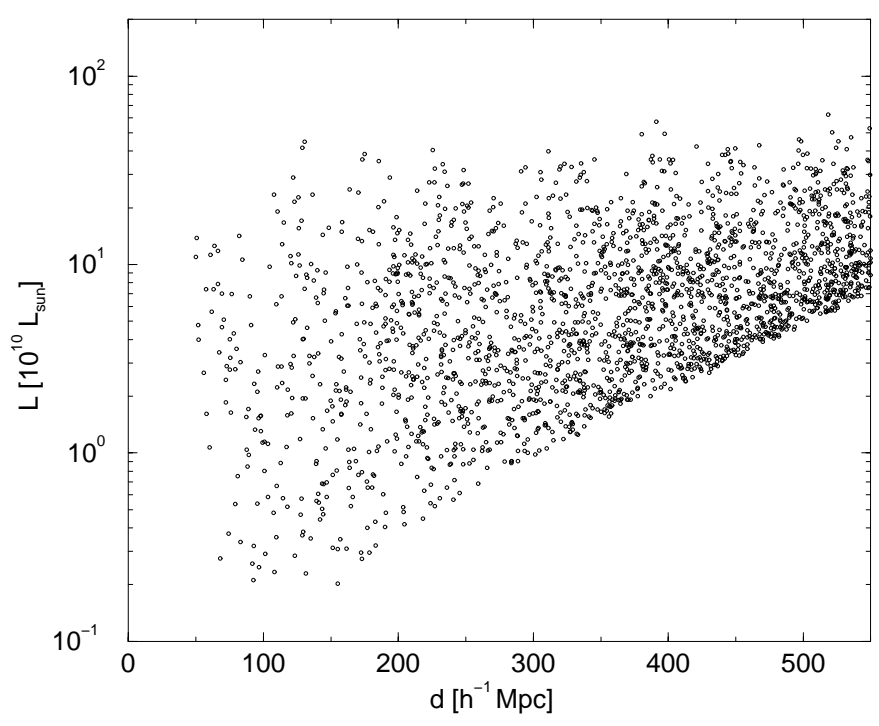

N2

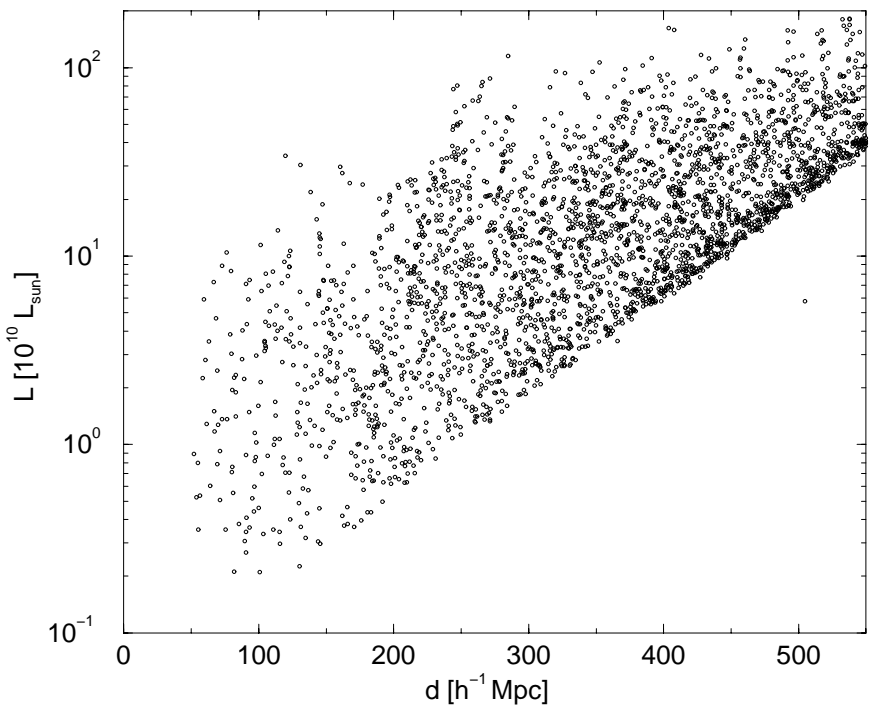

S2

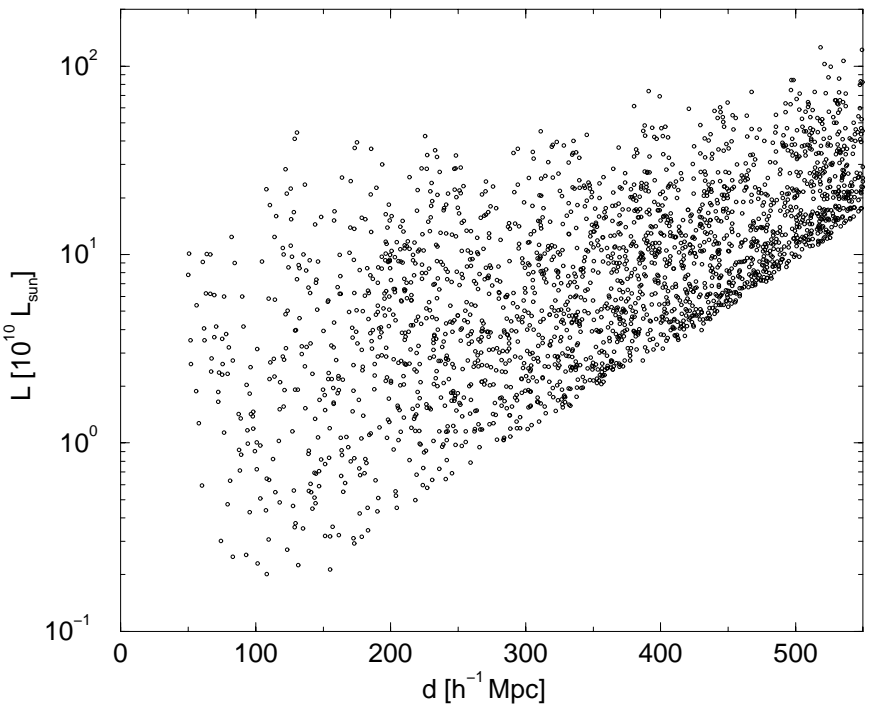

Fig. 6. Luminosities of DF-clusters as a function of distance. Upper panels shows the Northern slice, lower panels the Southern slice; left and right panels represent parameter set 1 and 2 , respectively.

distances from the observer, see left panels of Fig. 6. Schechter parameters of this set correspond to a subsample of galaxies in high-density environment, dominating in more distant regions. Using this set we find, that luminosities of most massive clusters also increase with distance. However, a small increase is expected since the volume of distant shells is larger, and thus the probability to find a bright galaxy in a shell of fixed thickness is higher on larger distance from the observer. Using the luminosity function derived in $\mathrm{H} 03$ we estimate that most luminous clusters near the outer border of the sample should be a factor of about 1.25 times more luminous than clusters in the middle of the sample. Figure 6 shows that this is the case for both the Northern and Southern slice for parameter set 1 . For this set of parameters the mean luminous matter density decreases with distance, as seen from Fig. 7.

The distributions of the mean density and of the cluster luminous masses are very sensitive tests for parameters of the luminosity function. Our tests show that it is impossible for one set of parameters to satisfy both criteria, the distance independence of the mean luminous density and that of the distribution of luminosities of clusters.

\section{Density field superclusters}

Superclusters have been traditionally defined either as clusters of clusters of galaxies (Oort 1983; Bahcall 1988), or as density enhancements in the galaxy distribution (de Vaucouleurs 1953; Saunders et al. 1991; Basilakos et al. 2001; Kolokotronis et al. 2002). In the first case Abell clusters were used to compile supercluster catalogues (E94, E97, E01). In the second case superclusters were found by using the distribution of individual galaxies or by using smoothed density field maps. Here we follow the density field approach and use the lowresolution density field to find large overdensity regions which 

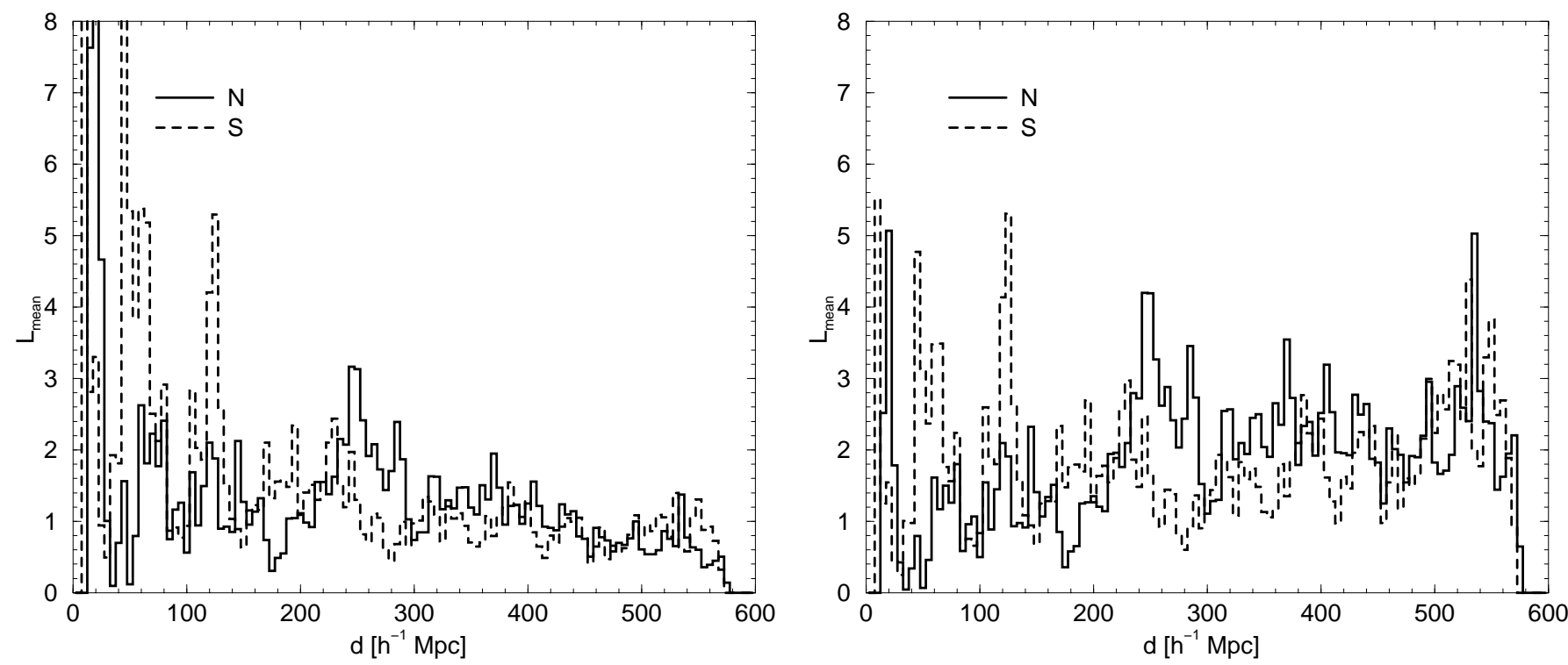

Fig. 7. The luminosity density in relative units of SDSS EDR Northern and Southern slices as a function of distance, for luminosity function parameters of set 1 (left) and 2 (right).

we call density field superclusters (or DF-superclusters). Tests with various smoothing lengths showed that a smoothing length $\sigma_{\mathrm{sm}}=10 h^{-1} \mathrm{Mpc}$ yields a catalogue of superclusters which matches well the catalogue of Abell superclusters. This length corresponds to the scale of the transition from the linear to the non-linear evolution. If we consider superclusters as systems in the linear regime of the evolution, then we must use for the smoothing a scale not smaller than the non-linearity scale. When a larger smoothing length is used then high-density regions tend to merge into larger aggregates, see Fig. 3.

The compilation of the supercluster catalogue consists of three steps: (1) calculating the density field, (2) finding overdensity regions, and (3) determining the properties of the resulting superclusters. The density field was calculated as described above with one difference - in order to reduce the wedge-like volume of slices to a sheet of uniform thickness we divided densities by the thickness of the slice at each particular distance. In this way the surface density of the field is on average independent of distance, and we can use a distance independent search for overdensity regions. The reduced density field for the Northern and Southern SDSS EDR slices is shown in Fig. 5.

Next we searched for connected high-density regions. To do so we need to fix the threshold density, $\varrho_{0}$, which divides high- and low-density regions. This threshold density plays the same role as the neighbourhood radius used in the friends-offriends (FoF) method to find clusters in galaxy samples and to find superclusters in cluster samples. If we have a high threshold density (this corresponds to a small neighbourhood radius), we get as connected regions only the central peaks of superclusters - the number of peaks is small, high-density regions cover only a small area, and their diameters are small. When we decrease the threshold density - corresponding to an increase in the neighbourhood radius in the FoF method - then the number of high-density regions increases and their areas and diameters also increase. When the threshold density is too low, then superclusters merge to form large complexes, their masses and diameters grow, and at a certain threshold density percolation occurs, i.e. the largest supercluster spans across the whole region under study.

To make a proper choice of the threshold density we plot in Fig. 8 the number of superclusters, $N$, the area of the largest supercluster $f$ (in units of the total area of the slice), and the maximum diameter of the largest supercluster (either in the $x$ or $y$ direction) as a function of the threshold density $\varrho_{0}$ (we use relative densities here). Data are given for both the Northern and the Southern slices. We see that the number of superclusters for the Northern slice has a broad maximum between $1.3<\varrho_{0}<$ 2.3, for the Southern slice the maximum is sharper at $\varrho_{0} \sim 1.5$. For lower $\varrho_{0}$ large superclusters are merged; for higher $\varrho_{0}$ fewer regions are counted as high-density. The area and diameter of the largest supercluster continue to drop rapidly until $\varrho_{0} \sim 2.0$. This shows that various criteria suggest different values for the threshold density. We have used a threshold density $\varrho_{0}=1.8$. At this threshold density the largest supercluster still has a diameter over $100 h^{-1} \mathrm{Mpc}$, it has several concentration centres (local density peaks), and its area forms a large fraction of the total area of the slice. The merging of large superclusters can be followed when we look for sudden changes in the relative area and diameter. For both slices superclusters are separated at $\varrho_{0}=2.1$; this density value is used to resolve the largest supercluster into sub-superclusters. Superclusters were found over the distance interval $100 \leq d \leq 550 h^{-1} \mathrm{Mpc}$. We include only superclusters with areas greater than $100\left(h^{-1} \mathrm{Mpc}\right)^{2}$; otherwise we get as superclusters tiny regions with diameters of less than $10 h^{-1} \mathrm{Mpc}$.

The number of superclusters in each of the two slices is shown in Table 1. In Table 3 we list for individual superclusters positional, physical, and morphological data. To aid in identifying these superclusters, we provide an identification number $N o$, the right ascension RA, the distance $d$, and rectangular coordinates, $x$, and $y$ (in $h^{-1} \mathrm{Mpc}$ ) used in the density field 


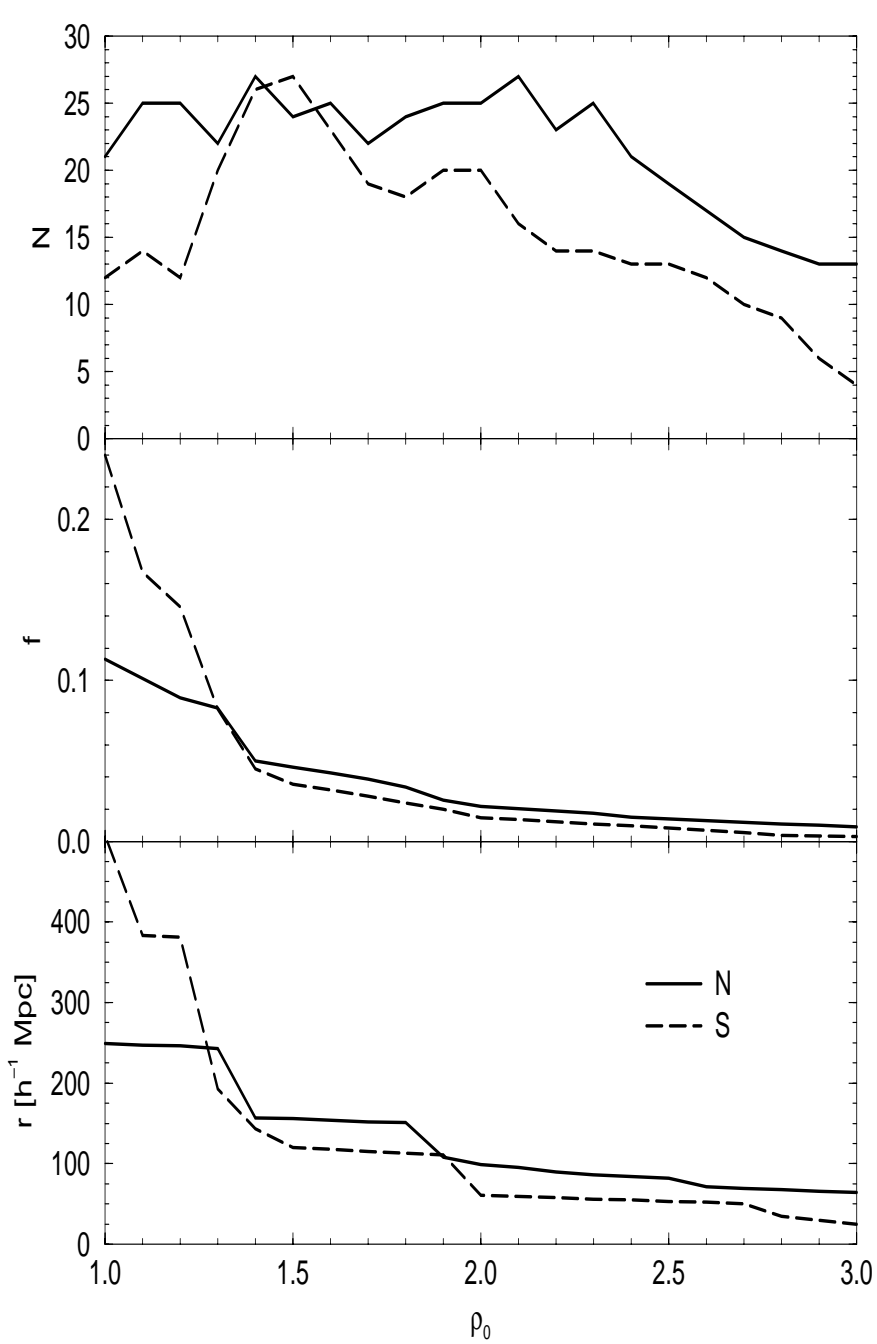

Fig. 8. Properties of SDSS EDR density field superclusters as a function of the threshold density, $\varrho_{0}$, to separate superclusters (highdensity regions) and voids (low-density regions). The upper panel shows the number of superclusters, $N$, the middle panel the area of the largest supercluster (in units of the total area of the slice), and the lower panel - the diameter (either in $x$ or $y$ direction, whatever is larger) of the largest supercluster.

plots. The column labelled "Id" gives the identification number from E01 of any matches to previously known superclusters based upon the Abell cluster sample.

For physical data we present the observed total luminosity of the supercluster $L_{\mathrm{obs}}$ (sum of observed luminosities of DF-clusters located within the boundaries of superclusters), the estimated total luminosity of the superclusters $L_{\text {tot }}$, the diameter $D$ of the supercluster (diameter of a circular area equal to the area of the supercluster), and $\Delta=\max (\mathrm{d} x, \mathrm{~d} y)$, where $\mathrm{d} x$ and $\mathrm{d} y$ are diameter of the supercluster in the $x$ and $y$ direction. The difference between the diameters $D$ and $\Delta$ yields information on the flattening of the supercluster in the plane of the slice. For round systems $D \sim \Delta$; for elongated superclusters, $\Delta$ exceeds $D$. The total luminosity $L_{\text {tot }}$ was calculated from the observed luminosity $L_{\mathrm{obs}}$

$L_{\mathrm{tot}}=\frac{D}{D_{\mathrm{d}}} L_{\mathrm{obs}}$, where $D_{\mathrm{d}}$ is the thickness of the slice at the distance of the centre of the supercluster, and we have assumed that the vertical diameter of the supercluster is identical to the diameter in the plane of the slice $D$. Finally, $f$ is the fraction of the area of the supercluster in units of the total area occupied by superclusters in a given slice.

For morphological data we give the number of DF-clusters and Abell clusters lying within the boundaries of the supercluster, $N_{\mathrm{cl}}$ and $N_{\mathrm{ACO}}$, respectively, see also Fig. 4 . We note that the number od Abell clusters corresponds to the number of main components of clusters, the number of secondary subclusters (if a cluster consists of several subclusters) is not given. Following is the type of the supercluster "T", estimated by visual inspection of the density field. Here we have used the following tentative classification: if the supercluster shows filamentary character, then its type is " $F$ " for a single filament or " $\mathrm{M}$ " for a system of multiple filaments. As an example of a filamentary system we refer to supercluster N04; an example of a multi-filamentary system is the supercluster N02. N04 consists of a single well-defined filament, and N02 of two massive filaments situated at almost right angles to each other. In both cases the main filaments are surrounded by a loose cloud of faint clusters. If such diffuse clusters dominate and the filamentary character is not evident, then the supercluster morphology is listed as a diffuse "D", an example being N08. Finally, "C" denotes a compact supercluster without clear filamentary system, an example being N15. This classification is based on the distribution of galaxies and clusters in a plane. If 3-dimensional data were available, then the true shape of each supercluster could be established.

It should be noted that most superclusters are surrounded by faint systems of galaxies, either in the form of filaments or of a diffuse cloud of clusters. Thus the distinction between a filamentary and diffuse system is not very strict.

\section{DF-clusters and superclusters as traces of the structure of the universe}

\subsection{Selection effects}

The main selection effect in the SDSS survey is due to the finite width of the apparent magnitude window, which excludes galaxies brighter or fainter than this window. The presence of a relatively narrow visibility window affects our results in two different ways. First of all, it influences the number and luminosity of galaxies in DF-clusters. This effect can be taken into account statistically in the determination of the luminosity of clusters using weighting of galaxies in DF-clusters. But the visibility window also affects the number of DF-clusters - a faint DF-cluster will not be visible at all at large distances if none of its member galaxies is bright enough to fall within the visibility window. The number of faint DF-clusters can be estimated statistically using the procedure described below in calculating the DF-cluster luminosity function. This procedure, however, cannot restore physical parameters or positions of individual invisible DF-clusters, similar to the first procedure which cannot restore parameters of invisible galaxies. 


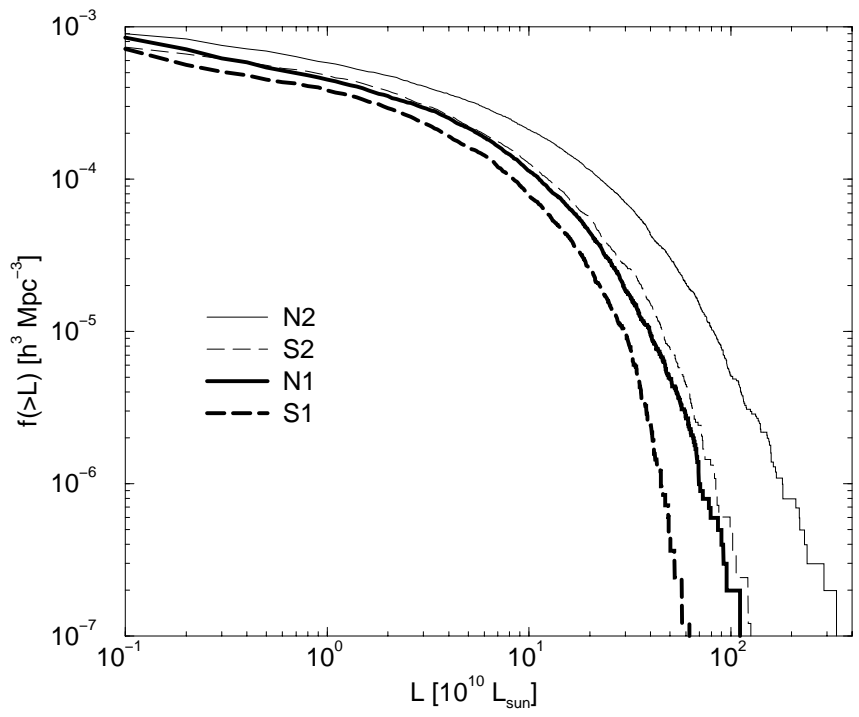

Fig. 9. Integrated luminosity function of DF-clusters for parameter sets 1 and 2 .

The weighting of visible galaxies in clusters statistically restores properties of clusters which have at least one galaxy in the visibility window of the survey. The procedure depends crucially on the parameters of the galaxy luminosity function which is used to derive the weights for the visible galaxies. We have used the constancy of the mean density with redshift to check the values for the luminosity function (parameter set 2). Our analysis has shown that the mean properties of superclusters are independent of the distance from the observer, which suggests that the global properties of the low-resolution density field are correct. However, in this case, the luminosities of the DF-clusters are too high at these large distances. The reason for the overcorrection of visible cluster luminosities is simple: visible clusters have to include also luminosities of invisible clusters. It is clear that in this case we get a wrong distribution of cluster luminosities. This is clearly seen in Fig. 4, where most distant clusters in both slices seem reddish (they have too high luminous density), whereas in nearby regions clusters have various colours from red to yellow, green and blue, representing clusters of various luminosity.

To avoid this effect we have used another set of values for the parameters of the galaxy luminosity function (parameter set 1 ). Now we get cluster luminosities which are, in the mean, independent of the distance from the observer. In this case the mean density of luminous matter decreases with distance, as do the mean luminosities of superclusters. This deficit of lowluminosity clusters can be estimated in the determination of the cluster luminosity function (see next section).

There are two possibilities to correct for the second selection effect in the calculation of the density field. We can add to the density field, calculated on the basis of the observed galaxies and corrected for unobserved galaxies associated with them, a smooth background which takes into account unseen galaxies not-associated with observed ones. In the calculation of the low-resolution density field this procedure yields the correct large-scale distribution of luminous matter - if the unseen galaxies are distributed randomly. However, this last assumption needs justification: faint galaxy systems, like bright systems, may be preferentially located within superclusters. On the other hand, this procedure also adds some luminous matter to DF-clusters, since luminous matter is collected into DF-clusters from the whole search box $\pm 2.5 h^{-1} \mathrm{Mpc}$ from the cluster centre. In other words, this correction method distorts DF-cluster properties. As we want to derive statistically correct cluster properties, we must use a high-resolution density field without the background correction. Taking into account these difficulties we have used a different method to find the low-resolution density field, by using for clusters and superclusters various parameters of the galaxy luminosity function. In Paper II we shall use a different method to find unbiased luminosities of DF-clusters.

\subsection{Luminosity function of DF-clusters}

Now we calculate the integrated luminosity function of DFclusters, i.e. the number of DF-clusters per unit volume exceeding a luminosity $L$. Figure 6 shows that only very bright DF-clusters are observable over the whole depth of our samples. These bright clusters form a volume limited subsample of DF-clusters. Fainter clusters are seen in the nearby volume only; the estimated total number of fainter clusters can be found by multiplying the observed number of clusters by a weighting factor of $\left(d_{\lim } / d_{\mathrm{L}}\right)^{3}$, where $d_{\mathrm{lim}}=550 \mathrm{~h}^{-1} \mathrm{Mpc}$ is the limiting distance used in compiling of the DF-cluster sample, and $d_{\mathrm{L}}$ is the largest distance where clusters of luminosity $L$ are seen. Figure 6 indicates that there exists an almost linear relation between $d_{\mathrm{L}}$ and $\log L$; this relation was used to calculate the weighting factor for clusters of every luminosity. Results for both SDSS samples are shown in Fig. 9.

We see that the luminosity functions span over 3 orders in luminosity and almost 4 orders in spatial density. Bright DF-clusters are density field equivalents of rich clusters of the Abell class, fainter DF-clusters correspond to groups of galaxies. The broad coverage of luminosities shows that the SDSS sample is well suited for determining the luminosity function from observations. Presently we have not determined masses of DF-clusters; thus it is not possible for us to transform the luminosity function to mass function of clusters. However, if we assume that the mass-to-luminosity ratio of DF-clusters is constant, then we can have an estimate of the DF-cluster mass function. Our luminosity function is not very different from the real observed mass function of conventional clusters and groups of galaxies (Ramella et al. 1997; Girardi \& Giuricin 2000; Heinämäki et al. 2003).

The integrated luminosity function was found for both parameters sets of the galaxy luminosity function (see Fig. 9). The number of bright clusters is much larger for the parameter set 2. As discussed above, this is due to the overcorrection of cluster luminosities - particularly at large distances - to compensate for non-detected faint clusters. Thus we believe that the function for parameter set 1 corresponds better to reality.

Our results also show that the Northern SDSS sample has a larger number of luminous DF-clusters than does the Southern sample. This difference is seen for both parameter sets of the 
$\mathrm{N}$

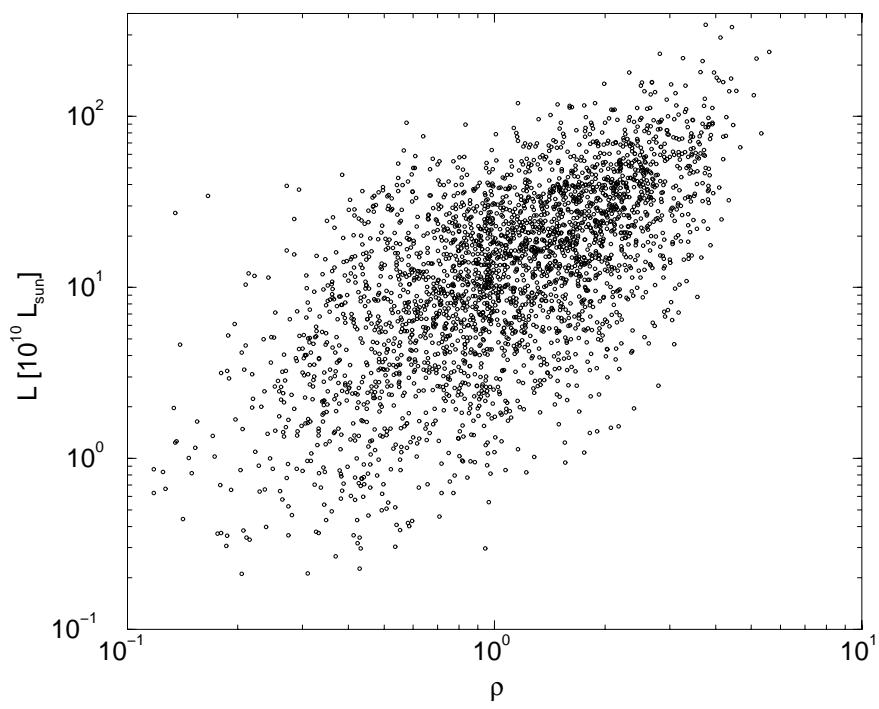

$S$

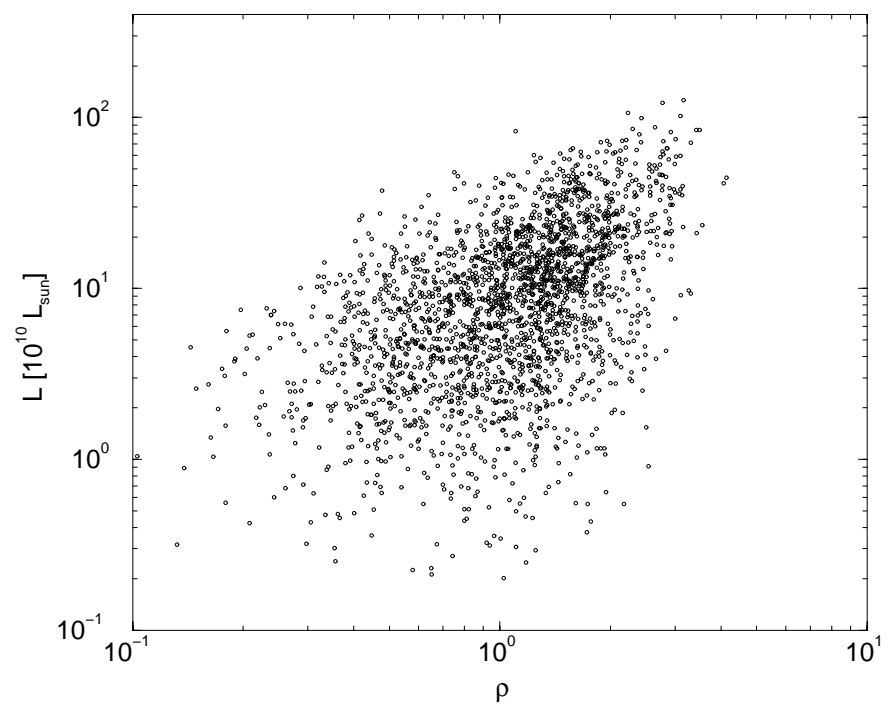

Fig. 10. Luminosities of DF-clusters as a function of the global relative density $\varrho_{0}$. Left panel shows the Northern slice, right panel the Southern slice.

galaxy luminosity function. In order to find the reason for this difference we repeated our calculations using different values for the parameters of the galaxy luminosity function for the Northern and Southern samples. This did not change the main result - there exists a considerable difference in the distribution of luminosities of DF-clusters in the Northern and Southern samples. The luminosity function of DF-clusters can be well approximated by the Schechter function, see Paper II.

\subsection{Environmental effects in the distribution of DF-clusters}

We can use the density found with the $10 h^{-1}$ Mpc smoothing as an environmental parameter to describe the global density in the supercluster environment of clusters. We calculated this global density $\varrho_{0}$ (in units of the mean density of the lowresolution density field) for all DF-clusters. Figure 10 shows the luminosity of DF-clusters as a function of the global density $\varrho_{0}$. There is a clear correlation between the luminosity of DF-clusters and their environmental density. Luminous clusters are predominantly located in high-density regions, and lowluminosity clusters in low-density regions. This tendency is seen also visually in Fig. 4. Here densities are colour-coded, we see that small clusters in voids have blue colour which indicates medium and small densities, whereas rich cluster having red colour populate dominantly central high-density regions of superclusters.

Figure 10 shows also that the Northern sample has much more luminous clusters than the Southern sample. This result confirms the presence of a difference between the structure of Northern and Southern samples.

Figure 11 shows the cluster luminosity functions of the Northern and Southern slice calculated separately for 4 global density intervals, $0<\varrho_{0} \leq 0.5,0.5<\varrho_{0} \leq 1.0,1.0<\varrho_{0} \leq 1.8$, and $1.8<\varrho_{0} \leq 10$, labelled in Fig. 11 and Table 2 as N0.0, N0.5, N1.0 and N1.8, for Northern subsamples, and S0.0, S0.5,
S1.0, S1.8 for Southern ones. The functions were calculated for the luminosity function parameter set 1 (which yields almost constant highest luminosity DF-clusters for various distances from the observer). We list in the Table 2 the number of DF-clusters in subsamples, $N_{\text {group }}$, the luminosity of mostluminous clusters, $L_{\mathrm{lum}}$, the mean luminosity of clusters, $L_{\mathrm{m}}$, and the scatter of luminosities, $\sigma\left(L_{\mathrm{m}}\right)$. We see that luminosity functions depend very strongly on the global environment: the difference in luminosity of most luminous clusters in subsamples is a factor of $5 \pm 2$ (for a finer global density division most luminous DF-clusters differ in luminosity even up to 10 in extreme density regions). This difference is not due to different numbers of clusters, as these numbers are all of the same order.

One may ask: How much of the cluster luminosityenvironment relation can be explained by the fact that a luminous cluster itself contributes to the luminosity density of the supercluster? At an extreme, suppose there is only one cluster in the supercluster. In that case, the supercluster's luminosity density is determined entirely by the single cluster. In reality, small scatter of the luminosity-environment relation at high environmental density is probably due just to proximity to luminous clusters themselves. This influence decreases when we move toward lower environmental densities. We plan to investigate this problem, as well as the influence of selection effects to the luminosity-density relationship of DF-clusters when more SDSS data will be available. The influence of selection is minimal to luminosities of most-luminous clusters.

\subsection{The fine structure of superclusters and voids}

The distribution of DF-clusters within DF-superclusters yields information on the internal structure of superclusters. A close inspection of Figs. 4 and 5 shows, that superclusters have various internal structures: clusters may form a single filament, a branching system of filaments, or a more or less diffuse cloud of clusters. These morphological properties have been 

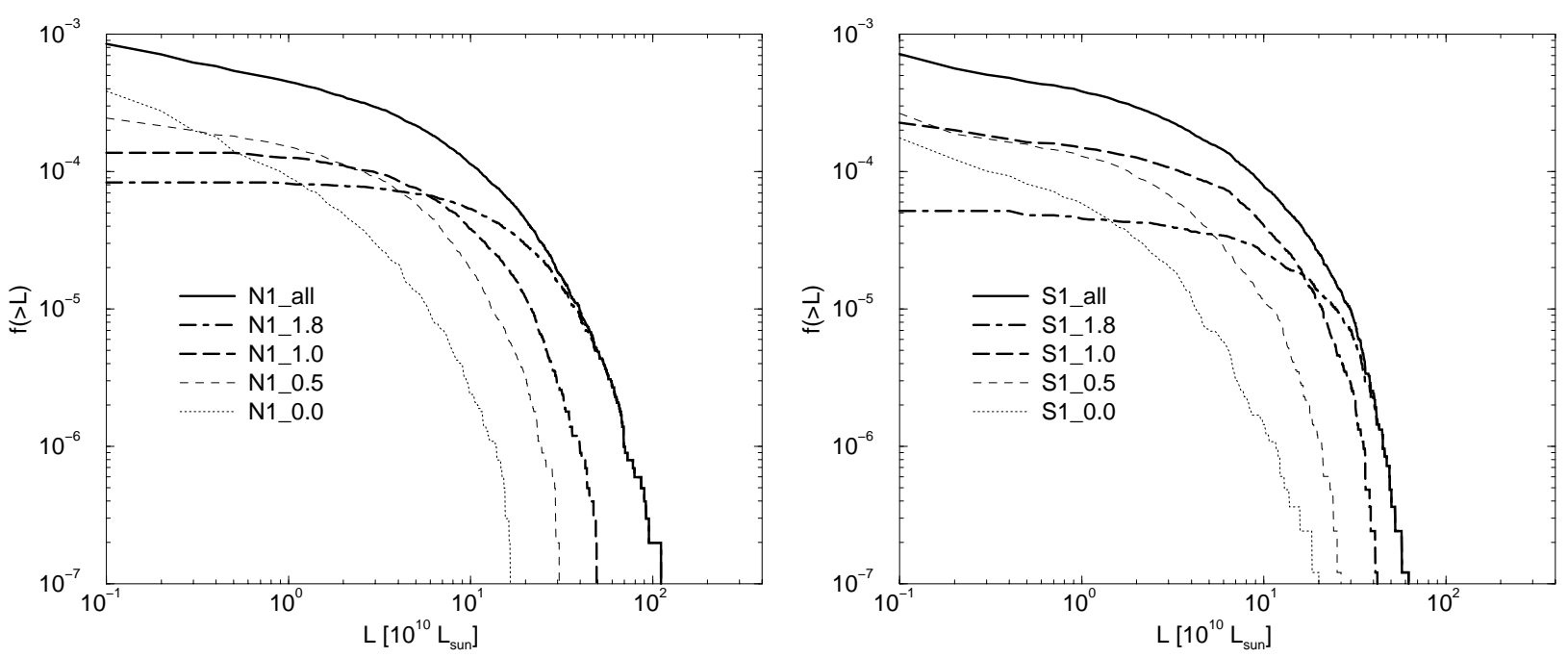

Fig. 11. Integrated luminosity functions of DF-clusters for various global relative density intervals $\varrho_{0}$. Left panel shows the Northern slice, right panel the Southern slice.

Table 2. Luminosities of DF-clusters.

\begin{tabular}{lcccc}
\hline \hline Sample & $N_{\text {group }}$ & $\begin{array}{c}L_{\text {lum }} \\
10^{10} L_{\odot}\end{array}$ & $\begin{array}{c}L_{\mathrm{m}} \\
10^{10} L_{\odot}\end{array}$ & $\begin{array}{c}\sigma\left(L_{\mathrm{m}}\right) \\
10^{10} L_{\odot}\end{array}$ \\
\hline N0.0 & 438 & 17.9 & 3.66 & 2.52 \\
N0.5 & 852 & 33.5 & 7.20 & 4.09 \\
N1.0 & 872 & 50.7 & 11.30 & 6.28 \\
N1.8 & 716 & 121.5 & 21.72 & 12.35 \\
& & & & \\
S0.0 & 277 & 20.0 & 3.57 & 2.15 \\
S0.5 & 695 & 26.5 & 5.60 & 3.30 \\
S1.0 & 965 & 42.1 & 9.57 & 5.48 \\
S1.8 & 341 & 62.3 & 16.93 & 9.84 \\
\hline
\end{tabular}

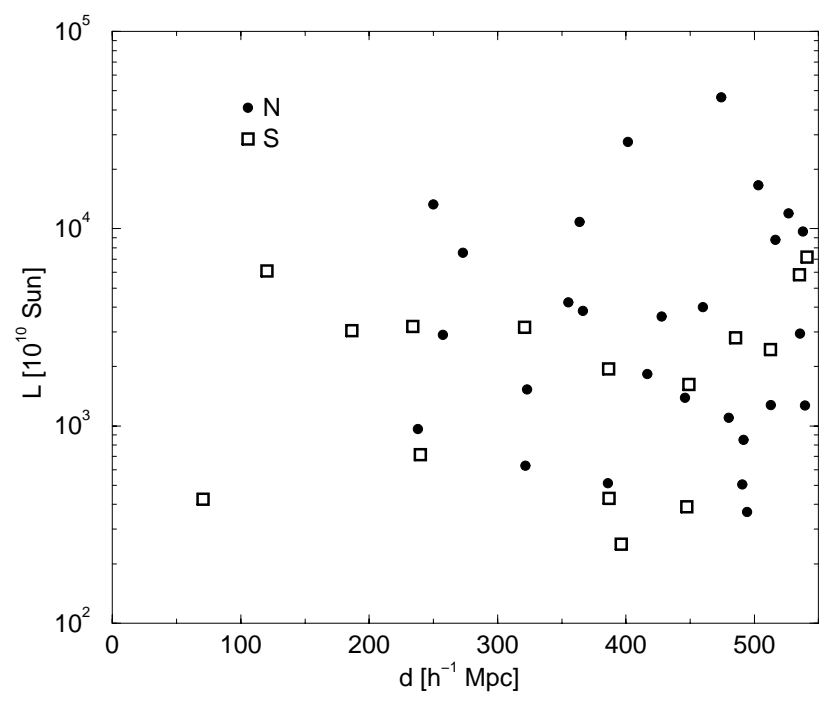

characterised by types F, M, D, and C in Table 3 . This table shows that almost all massive superclusters have morphological type M, i.e. they are multi-branching. Faint superclusters do not have a dominant morphological type: among faint superclusters we find all morphological types.

In the Northern sample about $25 \%$ of all DF-clusters are located in superclusters; in the Southern sample this fraction is about $15 \%$. An inspection of the high-resolution density map in Fig. 4 shows that most DF-clusters outside superclusters also form filaments. Thus we come to the conclusion that there is no major difference in the shape of cluster systems in supercluster and in non-supercluster environments. This similarity of the structure within and outside superclusters is partly due to our formal procedure of defining superclusters; actually there is a continuous sequence of structures from single filaments to multiple filaments and superclusters. The difference is mainly in the luminosity of clusters. This observation can be interpreted as follows: clusters and cluster filaments in various environments are formed by similar density perturbations, and these small-scale perturbations are modulated by large-scale perturbations which make clusters and their filaments richer in superclusters and poorer in large voids between superclusters (see also Frisch et al. 1995).

Fig. 12. Total luminosities of DF-superclusters in SDSS EDR slices at different distances from the observer.

Figure 12 shows the distribution of total luminosities of DF-superclusters at various distances from the observer. We see that both high and low luminosity superclusters are found both in nearby space as well as in more distant regions. This distribution is expected for real superclusters, and we may expect that there are no serious systematic errors in the luminosities of DF-superclusters.

Table 3 shows that there are only a few dozen Abell clusters in EDR SDSS sheets. Abell clusters form a small minority since only rich clusters were included into the Abell catalogue. In contrast DF-clusters were searched among galaxy systems of any richness. For this reason alone DF-clusters follow the fine distribution of luminous matter in much more detail than Abell clusters. We note that in some cases it is difficult to identify Abell clusters among DF-clusters, since there are several rich DF-clusters close in position to Abell clusters (see E03). Also, some Abell clusters consists of several subclusters; if such 
Table 3. The list of superclusters.

\begin{tabular}{|c|c|c|c|c|c|c|c|c|c|c|c|c|c|}
\hline$\overline{\mathrm{No}}$ & $\begin{array}{r}L_{\mathrm{obs}} \\
{\left[10^{10} L_{\odot}\right]}\end{array}$ & $\begin{aligned} & L_{\text {tot }} \\
& {\left[10^{10}\right.}\left.L_{\odot}\right]\end{aligned}$ & $\begin{array}{r}D \\
\mathrm{Mpc}\end{array}$ & $\begin{array}{r}\Delta \\
\mathrm{Mpc}\end{array}$ & $\begin{array}{l}\text { RA } \\
\text { deg }\end{array}$ & $\begin{array}{r}d \\
\mathrm{Mpc}\end{array}$ & $\begin{array}{r}x \\
M p c\end{array}$ & $\begin{array}{r}y \\
\mathrm{Mpc}\end{array}$ & $\overline{\overline{f f}}$ & $\overline{N_{\mathrm{cl}}}$ & 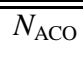 & 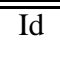 & $\overline{\mathrm{TT}}$ \\
\hline S01 & 186.6 & 714.1 & 24.0 & 35.0 & 1.2 & 239.7 & -90.5 & -222.0 & 0.0205 & 11 & 0 & 3 & $\bar{M}$ \\
\hline S02 & 554.2 & 1621.4 & 34.4 & 42.0 & 4.8 & 449.0 & -143.5 & -425.5 & 0.0420 & 20 & 0 & & M \\
\hline S03 & 345.6 & 3040.3 & 43.0 & 53.0 & 8.8 & 186.5 & -47.0 & -180.5 & 0.0655 & 30 & 2 & 19 & M \\
\hline S04 & 588.1 & 3164.9 & 45.2 & 63.0 & 9.2 & 320.9 & -79.0 & -311.0 & 0.0726 & 33 & 3 & 14 & M \\
\hline S05 & 283.7 & 642.6 & 32.8 & 36.0 & 9.7 & 552.4 & -131.5 & -536.5 & 0.0381 & 7 & 0 & & $\mathrm{~F}$ \\
\hline S06 & 159.7 & 251.8 & 16.4 & 23.0 & 13.2 & 396.2 & -70.0 & -390.0 & 0.0095 & 4 & 0 & & $\mathrm{~F}$ \\
\hline S07 & 59.8 & 121.7 & 29.6 & 33.0 & 15.1 & 554.8 & -80.0 & -549.0 & 0.0310 & 2 & 0 & & C \\
\hline S08 & 851.2 & 2441.7 & 38.5 & 45.0 & 19.2 & 512.4 & -37.5 & -511.0 & 0.0526 & 22 & 0 & & M \\
\hline S09 & 449.8 & 3187.9 & 43.4 & 59.0 & 23.9 & 234.0 & 2.0 & -234.0 & 0.0670 & 33 & 1 & 34 & M \\
\hline S10 & 379.0 & 6103.8 & 50.8 & 92.0 & 28.0 & 120.4 & 9.5 & -120.0 & 0.0915 & 29 & 1 & 24 & M \\
\hline S11 & 204.5 & 429.3 & 21.3 & 25.0 & 28.9 & 386.8 & 37.0 & -385.0 & 0.0161 & 5 & 0 & & M \\
\hline S12 & 955.7 & 2798.4 & 37.2 & 56.0 & 31.5 & 485.3 & 68.0 & -480.5 & 0.0492 & 24 & 0 & & M \\
\hline S13 & 1496.1 & 5852.5 & 54.8 & 65.0 & 43.2 & 535.0 & 181.0 & -503.5 & 0.1066 & 35 & 0 & & M \\
\hline S14 & 283.9 & 389.6 & 16.1 & 17.0 & 45.9 & 447.5 & 171.0 & -413.5 & 0.0092 & 7 & 0 & & M \\
\hline S15 & 518.9 & 1943.0 & 37.9 & 44.0 & 52.1 & 386.4 & 185.5 & -339.0 & 0.0510 & 24 & 0 & & M \\
\hline N01 & 2340.4 & 8754.9 & 50.6 & 85.0 & 150.6 & 516.4 & 331.5 & 396.0 & 0.0547 & 32 & 0 & & M \\
\hline N02 & 1027.0 & 7528.6 & 52.4 & 71.0 & 153.9 & 273.0 & 163.0 & 219.0 & 0.0587 & 36 & 4 & 82 & M \\
\hline N03 & 709.3 & 1271.4 & 25.3 & 32.0 & 158.6 & 539.3 & 285.5 & 457.5 & 0.0137 & 11 & 0 & & $\mathrm{~F}$ \\
\hline N04 & 875.1 & 3836.5 & 42.1 & 67.0 & 160.4 & 366.5 & 184.0 & 317.0 & 0.0379 & 26 & 0 & 252 & $\mathrm{~F}$ \\
\hline N05 & 1281.3 & 2943.2 & 32.2 & 36.0 & 166.9 & 535.4 & 214.5 & 490.5 & 0.0222 & 13 & 0 & & M \\
\hline N06 & 267.4 & 512.4 & 19.3 & 22.0 & 172.9 & 385.7 & 117.0 & 367.5 & 0.0080 & 6 & 0 & & $\mathrm{C}$ \\
\hline N07 & 228.8 & 628.6 & 23.1 & 26.0 & 178.6 & 321.5 & 66.5 & 314.5 & 0.0114 & 9 & 0 & 107 & $\mathrm{~F}$ \\
\hline N08 & 223.5 & 963.8 & 26.9 & 30.0 & 180.8 & 238.0 & 40.5 & 234.5 & 0.0154 & 11 & 0 & 111 & $\mathrm{D}$ \\
\hline N09 & 1833.0 & 10859.9 & 56.4 & 84.0 & 182.3 & 363.8 & 52.5 & 360.0 & 0.0681 & 64 & 2 & 265 & M \\
\hline N10 & 6412.0 & 46426.6 & 89.9 & 151.0 & 184.0 & 474.1 & 54.0 & 471.0 & 0.1728 & 116 & 0 & & M \\
\hline $\mathrm{N} 10 \mathrm{~A}$ & 701.1 & 1832.6 & 28.5 & 34.0 & 190.1 & 416.5 & 3.5 & 416.5 & 0.0257 & 20 & 0 & & M \\
\hline N10B & 1288.8 & 3994.9 & 37.3 & 40.0 & 185.6 & 459.7 & 39.5 & 458.0 & 0.0441 & 21 & 0 & & M \\
\hline N10C & 3616.2 & 16608.7 & 60.5 & 95.0 & 181.7 & 503.0 & 77.5 & 497.0 & 0.1158 & 54 & 0 & & M \\
\hline N11 & 306.5 & 367.4 & 15.5 & 16.0 & 185.8 & 494.2 & 41.5 & 492.5 & 0.0052 & 5 & 0 & & $\mathrm{D}$ \\
\hline $\mathrm{N} 12$ & 393.5 & 504.7 & 16.5 & 19.0 & 190.7 & 490.5 & -1.0 & 490.5 & 0.0058 & 7 & 0 & & $\mathrm{D}$ \\
\hline N13 & 1446.1 & 13240.7 & 59.9 & 90.0 & 198.3 & 249.8 & -33.5 & 247.5 & 0.0767 & 56 & 1 & 126 & M \\
\hline N14 & 2704.0 & 11938.6 & 60.9 & 78.0 & 198.5 & 526.5 & -72.5 & 521.5 & 0.0793 & 50 & 0 & & $\mathrm{D}$ \\
\hline N15 & 428.0 & 1533.3 & 30.3 & 32.0 & 200.2 & 323.0 & -54.0 & 318.5 & 0.0196 & 12 & 0 & & $\mathrm{C}$ \\
\hline N16 & 1115.7 & 3590.8 & 36.0 & 40.0 & 207.3 & 427.7 & -123.5 & 409.5 & 0.0278 & 25 & 0 & & $\mathrm{D}$ \\
\hline N17 & 571.3 & 1101.2 & 24.2 & 25.0 & 209.9 & 480.1 & -159.0 & 453.0 & 0.0126 & 13 & 0 & & $\mathrm{D}$ \\
\hline N18 & 2532.5 & 9667.2 & 53.7 & 69.0 & 212.1 & 537.6 & -197.5 & 500.0 & 0.0618 & 34 & 0 & & M \\
\hline N19 & 443.6 & 847.8 & 24.6 & 28.0 & 215.3 & 491.5 & -205.5 & 446.5 & 0.0129 & 8 & 0 & & $\mathrm{C}$ \\
\hline N20 & 3738.6 & 27566.5 & 77.5 & 94.0 & 216.8 & 401.4 & -177.5 & 360.0 & 0.1285 & 102 & 2 & & M \\
\hline $\mathrm{N} 21$ & 656.5 & 1274.0 & 26.1 & 33.0 & 220.1 & 512.8 & -253.0 & 446.0 & 0.0145 & 10 & 0 & & $\mathrm{C}$ \\
\hline $\mathrm{N} 22$ & 536.3 & 2891.5 & 36.4 & 57.0 & 230.0 & 257.6 & -163.5 & 199.0 & 0.0283 & 16 & 1 & 153 & M \\
\hline $\mathrm{N} 23$ & 928.3 & 4221.6 & 42.3 & 52.0 & 230.4 & 355.0 & -227.5 & 272.5 & 0.0382 & 32 & 1 & 155 & M \\
\hline N24 & 595.3 & 1387.1 & 27.2 & 28.0 & 233.1 & 445.9 & -301.5 & 328.5 & 0.0158 & 13 & 0 & & M \\
\hline S16 & 1572.5 & 7159.3 & 64.5 & 113.0 & 359.1 & 541.1 & -223.0 & -493.0 & 0.1478 & 45 & 0 & & M \\
\hline
\end{tabular}

Distance, sizes and coordinates are given in $h^{-1} \mathrm{Mpc}$.

subclusters have comparable number of members, then in Fig. 4 all subclusters are marked. For this reason the number of Abell clusters marked in this figure is larger than given in Table 3, where each Abell cluster is counted once.

\subsection{DF-superclusters in the supercluster-void network}

Now we analyse DF-superclusters as tracers of the large-scale supercluster-void network. First we note that most nearby
DF-superclusters can be identified with the Abell supercluster catalogue of E01, which contains superclusters out to a distance limit of $350 \mathrm{~h}^{-1} \mathrm{Mpc}$ (see Table 3 and Fig. 5). Now we comment on some individual superclusters.

The supercluster N13 (SCL 126 in the list by E01) has the densest concentration of Abell and X-ray clusters in our neighbourhood. The densest part of this supercluster has a declination $-2.5^{\circ}$ and lies in the $-3^{\circ}$ slice of the Las Campanas Redshift Survey. It contains 7 Abell clusters and 6 X-ray 


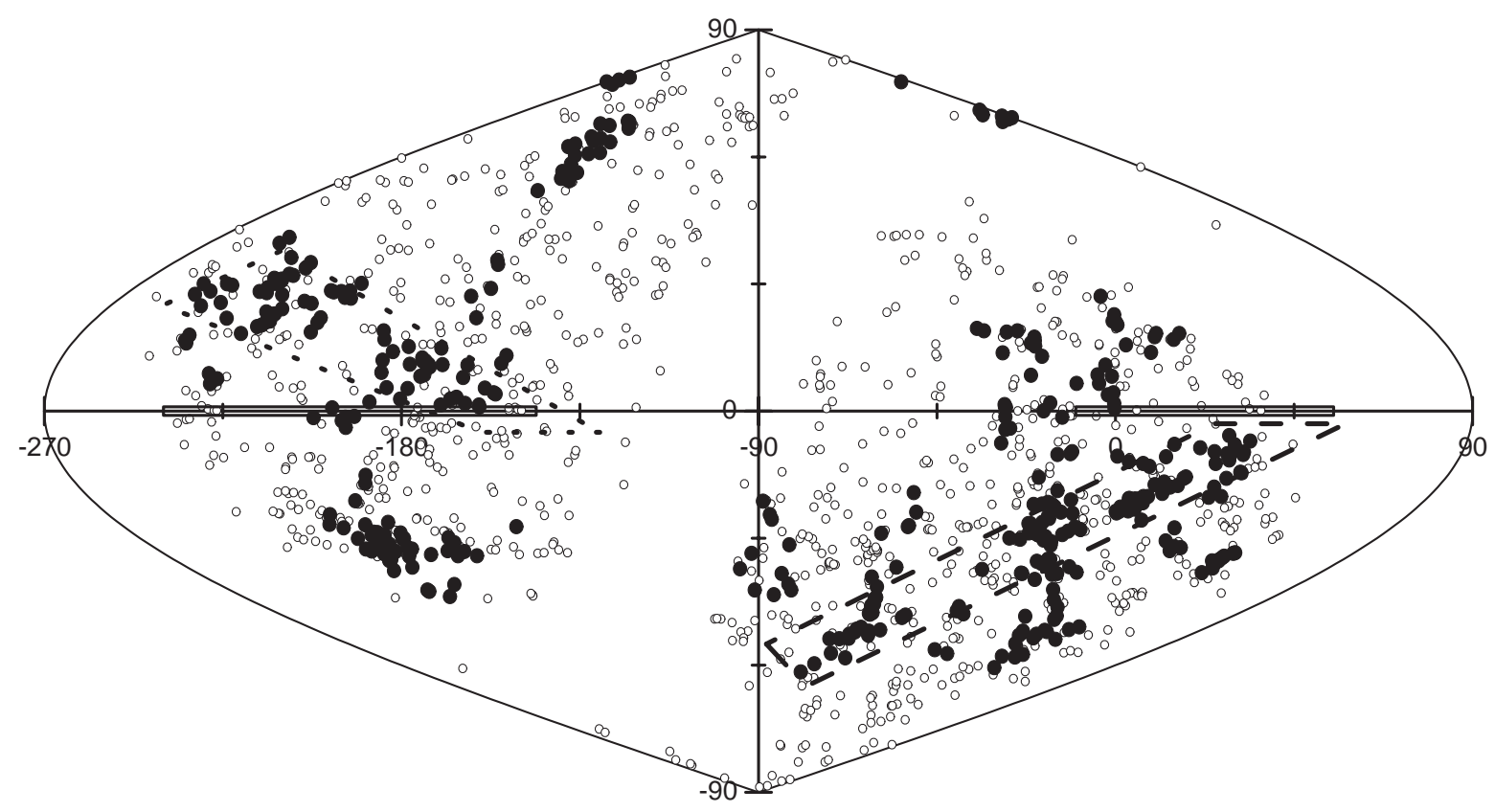

Fig. 13. A view to Abell clusters in equatorial coordinates. Filled circles show Abell clusters located in superclusters of richness 8 and more members, open circles mark Abell clusters in less rich superclusters. Strips near the celestial equator note SDSS slices, the Northern slice is in the left side. Areas surrounded by dashed lines indicates regions where rich superclusters of the Dominant Supercluster Plane are located. The Galactic zone of avoidance has in equatorial coordinates a S-shaped curve.

clusters, most of which are located within the LCRS slice. Both in the SDSS and LCRS slices the supercluster shows a multi-branching filamentary shape. The form of the filaments in both slices is different. This is a good indication that cluster chains form true filaments, not sheets, since at the distance of the supercluster both slices are vertically shifted by only about $12 h^{-1}$ Mpc.

Another very rich supercluster is N08 (Virgo-Coma; SCL 111 in E01's list). This supercluster contains 15 Abell clusters and $5 \mathrm{X}$-ray clusters. Its centre lies at declination $+9^{\circ}$; thus the SDSS slice passes through the outskirts of this supercluster. Not far from E01 SCL 111 lies the supercluster SCL 91 in the list by E97 (Leo-Sextans); it contains 9 Abell clusters and 1 $\mathrm{X}$-ray cluster at declination $+3^{\circ}$. This supercluster is seen as a density enhancement in Fig. 5; its density in the SDSS slice is, however, too low to include this feature as a supercluster in the present catalogue.

There are two more very rich superclusters in the Northern slice: SCL 107 from E97 (N07) with 8 Abell clusters and 1 Xray cluster, and SCL 100 from E97 with 9 Abell clusters. Their centres lie at declinations $+10^{\circ}$ and $-3^{\circ}$, respectively, and they form together with supercluster SCL 111 (N09) a huge complex near the centre of the SDSS Northern slice. These superclusters, and superclusters SCL 82 and SCL 91 belong to the Dominant Supergalactic Plane - a collection of very rich superclusters in the nearby Universe (E97).

The richest supercluster in the Southern slice, according to Abell supercluster catalogue, is the Pegasus-Pisces SCL 3 from E97 (or S01 in our catalogue) with 9 Abell clusters, 6 Xray clusters, and a centre at declination $+5^{\circ}$. The second richest supercluster in the Southern slice is the Pisces SCL 24 (S10) with 7 Abell clusters and 10 X-ray clusters and a centre at declination $+8^{\circ}$. The SDSS Southern slice passes through the periphery of these superclusters.

\subsection{The North-South asymmetry in the distribution of clusters and superclusters}

One of principal results of our study is the discovery of a large difference between the distribution of galaxies and clusters in the Northern and Southern slice of the SDSS. A moderate difference is observed in the galaxy luminosity functions, a much larger difference exists between distributions of cluster luminosities and between cluster luminosity functions (see Figs. 9, 10, and 11). The North-South asymmetry is seen also as a systematic difference of total luminosities of Northern and Southern DF-superclusters: the Northern sample contains more massive superclusters than the Southern one (see Table 3 and Fig. 12). These results indicate the presence of global differences between the Northern and Southern slices.

One possibility to explain these differences in the distribution of clusters and superclusters is by various parameters of the galaxy luminosity function. To check this possibility we varied parameters of the luminosity function of galaxies in accordance with results obtained in H03. In all variants tested so far the North-South asymmetry remains. Thus the difference lies probably elsewhere.

Another explanation would be to assume that these differences can be caused by cosmic variance. To check this possibility we compared properties of various systems of galaxies in the Northern and Southern slices. This has lead us to conclude that the most likely explanation of the North-South asymmetry is the presence of real differences in the richnesses of systems of galaxies of various scales. As we have discussed in the 
previous section, the Northern slice lies in a region of space containing many rich superclusters. The $\pm 10^{\circ}$ zone around the celestial equator in the right ascension interval of the Northern slice contains 25 Abell superclusters in the catalogue by E01 ( 15 in the $\pm 6^{\circ}$ zone). Among these, there are 5 very rich superclusters containing 7 or more Abell clusters (all in the central $\pm 6^{\circ}$ zone). The $\pm 10^{\circ}$ zone around the Southern slice has 12 Abell superclusters; the $\pm 6^{\circ}$ zone has 7éAbell superclusters, among which there is only one very rich system. The distribution of Abell clusters on the celestial sphere is presented in Fig. 13. Regions of the SDSS EDR are marked by solid strips, dashed lines show regions where rich superclusters belonging to the Dominant Supergalactic Plane (DSP) are projected on the sky. The DSP was detected by E97. In this plane, there are concentrated numerous very rich superclusters. We see that the Northern SDSS EDR slice crosses the DSP. Near the supercluster SCL 126 (or N13 in our list) the DSP crosses another region of rich superclusters seen in the Northern hemisphere extending from a declination of $+60^{\circ}$ to a declination of $-30^{\circ}$. So the Northern slice lies at crossroads of two complexes of very rich superclusters. In contrast, the Southern slice is located in a region away from very rich superclusters: in the Southern Hemisphere, rich superclusters, some of which belong to the DSP, are located at lower declinations. What concerns galaxies, then Gaztañaga (2002) has found no statistical difference in their photometric properties in both hemispheres.

Thus our tentative conclusion is that the North-South asymmetry is not a mere random fluke but a real difference in the large-scale distribution of matter. So far this difference is manifested in only a single pair of slices. This result must be checked by future SDSS data.

\subsection{Error analysis}

There are three main error sources in our quantitative data: errors due to number statistics (Poisson errors), errors in the values for the parameters of the galaxy luminosity function, and errors in our assumptions. Our main assumptions were: absence of projection effects in the $z$-direction, the luminosity function can be applied to individual clusters, the mean luminous density and the upper end of luminosities of DF-clusters do not depend on distance. Our quantitative analysis is related directly to luminosities of DF-clusters. Thus we must analyse how these errors affect cluster luminosities.

The simplest errors are related to the Poisson statistics. In H03 we determined Poisson errors for parameters of the luminosity function of galaxies. It is easy to estimate the influence of these errors to properties of density field clusters. We have repeated the density field analysis with many sets of parameters. We show here results for a third set of parameters $M^{\star}=-20.73$ and $\alpha=-1.14$, the difference between sets 2 and 3 being $\Delta M^{\star}=0.07$, and $\Delta \alpha=0.08$, which corresponds approximately to the $2 \sigma$ error of parameters in the luminosity function (see H03). We have found DF-clusters for both parameter sets and made cross-identification of clusters. Cluster positions are practically identical, only total luminosities differ. Both luminosities are compared in Fig. 14. We see that there

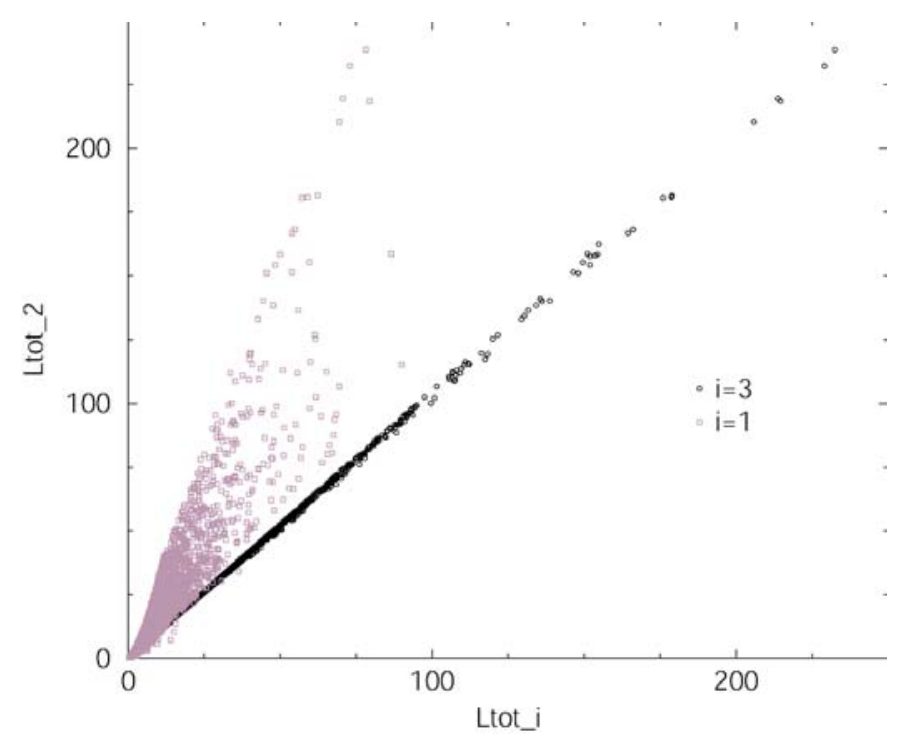

Fig. 14. Comparison of luminosities of DF-clusters for different parameter sets. In vertical axis we plot luminosities of clusters for parameter set 2, in horizontal axis either for set 1 or 3 .

exists a very close relationship with small scatter between luminosities of DF-clusters found with parameter sets 2 and 3. Our main quantitative results (North-South asymmetry of luminosities and dependence of luminosities on environment) are differential, thus errors of parameters of the luminosity function of galaxies do not alter these conclusions.

A much more serious systematic difference is due to our poor understanding of the global behaviour of the mean luminosity density, i.e. the third error mentioned above. The influence of this error is also demonstrated in Fig. 14, where we compare luminosities of DF-clusters for parameter sets 1 and 2. Here the scatter is much larger - almost all clusters are much brighter for the parameter set 2 . This is the reason why cluster luminosity functions for parameter sets 1 and 2 differ so much. The physical reason for this large uncertainty is our poor understanding of the general behaviour of the density field - how constant it should be for different distances from the observer. We plan to come back to this problem in a future study.

To check the possible error due to the use of 2-dimensional density field we calculated at all cluster positions the distribution of galaxies in $z$-direction. In the vast majority of cases there is only one maximum, or positions of maxima differ by $\leq 3 h^{-1} \mathrm{Mpc}$, which corresponds to the resolution in the $x, y$-plane. However, in about $4 \%$ of clusters there are several density maxima at cluster positions which differ in vertical position $\geq 4 h^{-1} \mathrm{Mpc}$. Due to this error total luminosities of these clusters are overestimated, and a similar number of poor DF-clusters is absent from our list. This error is partly compensated by another error: clusters near the border of the slice may be cut by borders, thus their luminosities are underestimated. Both errors can be avoided when using full 3-dimensional data, which will be done in the next stage of our study.

To determine the scatter of DF-cluster luminosities due to the scatter of galaxy luminosities in individual clusters we use numerical simulations. Results of this study shall be published elsewhere. 
There are other sources of uncertainty, ones which influence positions of DF-clusters and superclusters. These errors are due to the fact that in this paper we have used positions of galaxies in redshift space. Redshift space distortions are of two different types: they elongate clusters of galaxies in the radial direction (finger-of-god effect), and they shift positions of DF-clusters and superclusters in the radial direction toward the contraction centre (bulk motions). As superclusters are more massive attractors than clusters, their positions are much less influenced than are positions of clusters. Due to bulk motions supercluster shapes are slightly compressed in the radial direction. The shift is small and does not influence luminosities of clusters and superclusters.

The largest uncertainty in the properties of superclusters is due to the fact that we presently have information only for thin slices. In their largest dimension, a supercluster may extend for hundreds of $\mathrm{Mpc}$; this is much, much larger than the smallest dimension of our slices, their thickness. Therefore, it is impossible to say for certain just what fraction of a given supercluster lies within a slice's boundaries. Our estimate of the total luminosity of superclusters is based on the assumption that they have in vertical direction a structure similar to the structure on the plane observed. This assumption is correct if the observed plane passes close to the centre of the supercluster. If the observed plane passes through the periphery of the supercluster, we get an underestimated value of the total luminosity and diameter. Thus values shown in Table 3 can be considered as lower limits of actual values of the respective quantities.

\section{Discussion and conclusions}

Results of this paper and $\mathrm{H} 03$ are of a methodical and quantitative character. Methodical aspects concern the application of the density field method with various smoothing lengths to display and describe systems of galaxies of various scales. We calculated density fields for a number of smoothing lengths from 0.8 to $16 h^{-1} \mathrm{Mpc}$. In this way systems of galaxies on various scales could be visualised and their mutual relationship could be studied. The high-resolution density field, with dispersion $0.8 h^{-1} \mathrm{Mpc}$ was used to define density field clusters and to investigate the structure of superclusters. The low-resolution density field, with dispersion $10 h^{-1} \mathrm{Mpc}$ was used to define superclusters of galaxies and to study global properties of clusters and superclusters.

The inspection of the distribution of clusters in superclusters and voids suggests that galaxy systems have in both regions similar shape in that the dominant structural elements are single or multi-branching filaments. Massive superclusters have dominantly a multi-branching morphology; less massive superclusters have various morphologies, including compact, filamentary, multi-branching, and diffuse systems.

Quantitative results concern the luminosity function of galaxies and properties of clusters and superclusters. We have found in $\mathrm{H} 03$ and confirmed in this paper that it is impossible to find a global set of parameters of the luminosity function which can be applied in all cases. We found that parameters of the luminosity function are different for high- and low-density regions: galaxies in high-density regions are more luminous. This result confirms earlier findings by Lindner et al. (1995): bright galaxies define larger voids than faint ones. More recently this conclusion was obtained by Bromley et al. (1998), Beisbart \& Kerscher (2000), and by Norberg et al. (2001). The present study indicates that the effect is larger than previously suspected: luminosities of the brightest galaxies in high-density regions exceed the luminosities of the brightest galaxies in low-density regions by a factor of about 5 .

The values of the parameters of the luminosity function are also different for nearby and distant parts of the survey and for the Northern and Southern slices. A distance dependence of parameters of the luminosity function has been found for deeper surveys spanning redshift interval up to $z=1$ by Lin et al. (1999) and by Shepherd et al. (2001) in the Canadian Network for Observational Cosmology Redshift Survey. However, in this survey the distance dependence appears only by comparison of nearby $(z \sim 0)$ and distant $(z>$ $0.2)$ parts of the survey. Thus it seems improbable that this effect can explain our results on the distance dependence in a relatively small redshift interval $0<z \leq 0.2$.

Parameters of the luminosity function depend also on the density of the environment. This dependence influences properties of the density field. High-density regions contain brighter galaxies than do low-density ones. This difference leads to different selection effects for galaxies in high- and low-density environments. In a high-density environment, due to selection effects, faint galaxies in clusters cannot be observed, but clusters themselves are visible (since they contain at least one galaxy bright enough) and the total luminosity of clusters can be estimated by taking into account unobserved galaxies. In a low-density environment all galaxies of the cluster may be too faint, and the cluster may not be seen at all. In another words, distance-dependent selection effects influence clusters and superclusters in different ways.

The density field was calculated using the expected total luminosities of clusters of galaxies, including the expected luminosities of galaxies too faint or too bright to be included in the redshift survey. The correction for unobserved galaxies was made assuming a Schechter luminosity function for galaxies. Our analysis shows that it is impossible to correct the density field so that general properties of the density field and properties of clusters of galaxies are correct for the same set of parameters of the galaxy luminosity function. For this reason we have used two sets of parameters of the luminosity function. Parameter set 1 has a strong bright-end (parameter $\left.M^{\star}=-21.55\right)$ and corresponds to galaxies in a high-density environment which dominate clusters observed at large distance; this set yields correct properties of clusters of galaxies, but does not include faint clusters at large distances, and thus gives too low of a density for distant superclusters. Parameter set 2 was obtained for the whole region under study, and it has moderate bright-end (parameter $M^{\star}=-20.80$ ). In this parameter set ALL faint invisible galaxies at large distance are included within visible clusters, including galaxies that belong to non-detected clusters; thus clusters themselves become too luminous with increasing distance, but supercluster properties are correct. 
Comparing clusters in different environments we have found that there exists a strong dependence of cluster properties on the density of the large-scale environment: clusters located in high-density environments are a factor of $5 \pm 2$ more luminous than clusters in low-density environments, in extreme environmental density regions the difference is even larger.

Finally we found that there exists a large difference between properties of clusters and superclusters in the Northern and Southern slices of the SDSS EDR survey: clusters and superclusters in the Northern slice are more luminous than those in the Southern slice by a factor of 2 . This difference may be due to differences in the location of slices with respect to the very large-scale environment. If this conclusion is confirmed by future observations one must conclude that the formation and evolution of galaxies and systems of galaxies of various scales depends on the nearby as well as on the large-scale environment. Richer superclusters have more luminous galaxies and clusters. On smaller scales this tendency has been observed as a difference between properties of clusters within superclusters and in voids. Now we see that a similar difference may be observed on much larger scales.

Acknowledgements. We thank Heinz Andernach and Erik Tago for the permission to use their new compilation of redshifts of Abell clusters. The present study was supported by Estonian Science Foundation grants ETF 2625, ETF 3601, ETF 4695, and TO 0060058S98. P.H. was supported by the Finnish Academy of Sciences. J.E. thanks Fermi-lab and Astrophysikalisches Institut Potsdam (using DFG-grant 436 EST 17/2/01) for hospitality where part of this study was performed.

Funding for the creation and distribution of the SDSS Archive has been provided by the Alfred P. Sloan Foundation, the Participating Institutions, the National Aeronautics and Space Administration, the National Science Foundation, the U.S. Department of Energy, the Japanese Monbukagakusho, and the Max Planck Society. The SDSS Web site is http://www. sdss.org/.

The SDSS is managed by the Astrophysical Research Consortium (ARC) for the Participating Institutions. The Participating Institutions are The University of Chicago, Fermi-lab, the Institute for Advanced Study, the Japan Participation Group, The Johns Hopkins University, Los Alamos National Laboratory, the Max-Planck-Institute for Astronomy (MPIA), the Max-Planck-Institute for Astrophysics (MPA), New Mexico State University, University of Pittsburgh, Princeton University, the United States Naval Observatory, and the University of Washington.

\section{References}

Abell, G. 1958, ApJS, 3, 211

Abell, G., Corwin, H., \& Olowin, R. 1989, ApJS, 70, 1

Andernach, H., \& Tago, E. 1998, in Large Scale Structure: Tracks and Traces, ed. V. Müller, S. Gottlöber, J. P. Mücket, \& J. Wambsganss (Singapore: World Scientific), 147

Bahcall, N. 1988, ARA\&A, 26, 631

Basilakos, S. 2003, MNRAS, submitted [astro-ph/0302596]

Basilakos, S., Plionis, M., \& Rowan-Robinson, M. 2001, MNRAS, 323,47

Beisbart, C., \& Kerscher, M. 2000, ApJ, 545, 6

Bertschinger, E., Dekel, A., Faber, S. M., Dressler, A., \& Burstein, D. 1990, ApJ, 364, 370
Bromley, B. C., Press, W. H., Lin, H., \& Kirshner, R. P. ApJ, 505, 25 Carlberg, R. G., Yee, H. K. C., \& Ellingson, E. 1997, ApJ, 478, 462

Davis, M., \& Huchra, J. 1982, ApJ, 254, 437

Dalton, G. B., Maddox, S. J., Sutherland, W. J., \& Efstathiou, G. 1997, MNRAS, 289, 263

de Vaucouleurs, G. 1953, MNRAS, 113, 134

Einasto, J., Einasto, M., Hütsi, G., et al. 2003b, A\&A, in preparation, Paper II

Einasto, M., Einasto, J., Müller, V., Heinämäki, P., \& Tucker, D. L. 2003a, A\&A, 401, 851 [astro-ph/0211590] (E03)

Einasto, M., Einasto, J., Tago, E., Dalton, G., \& Andernach, H. 1994, MNRAS, 269, 301 (E94)

Einasto, M., Einasto, J., Tago, E., Müller, V., \& Andernach, H. 2001, AJ, 122, 2222 (E01)

Einasto, M., Tago, E., Jaaniste, J., Einasto, J., \& Andernach, H. 1997, A\&AS, 123, 119 (E97)

Frisch, P., Einasto, J., Einasto, M., et al. 1995, A\&A, 296, 611

Gaztañaga, E. 2002, ApJ, 580, 144

Girardi, M., \& Giuricin, G. 2000, ApJ, 540, 45

Goto, T., Okamura, S., McKay, T. A., et al. 2002, PASJ, 54, 515

Gott, J. R., Melott, A. L., \& Dickinson, M. 1986, ApJ, 306, 341

Heinämäki, P., Einasto, J., Einasto, M., et al. 2003, A\&A, 397, 63 [astro-ph/0202325]

Hoyle, F., Vogeley, M. S., Gott, J. R., et al. 2002, ApJ, 580, 663 [stro-ph/0206146]

Huchra, J., Davis, M., Latham, D., \& Tonry, J. 1983, ApJS, 52, 89

Hütsi, G., Einasto, J., Tucker, D. L., et al. 2003, A\&A, submitted (H03) [astro-ph/0212327]

Kaiser, N. 1984, ApJL, 284, L9

Kim, R. S. J., Kepner, J. V., Postman, M., et al. 2002, AJ, 123, 20

Kolokotronis, V., Basilakos, S., \& Plionis, M. 2002, MNRAS, 331, 1020

Lin, H., Yee, H. K. C., Carlberg, et al. 1999, ApJ, 518, 533

Lindner, U., Einasto, J., Einasto, M., et al. 1995, A\&A, 301, 329

Lineweaver, C. H., Tenorio, L., Smoot, G. F., et al. 1996, ApJ, 470, 38

Marinoni, C., Giuricin, G., \& Ceriani, L. 1999, Proceedings of the 1st Workshop of the Italian Network "Formation and Evolution of Galaxies", 4

Moore, B., Frenk, C. S., \& White, S. D. M. 1993, MNRAS, 261, 827

Norberg, P., Baugh, C. M., Hawkins, E., et al. 2001, MNRAS, 328, 64

Oort, J. H. 1983, ARA\&A, 21, 373

Peacock, J. A. 1999, Cosmological Physics (Cambridge: Cambridge University Press)

Ramella, M., Pisani, A., \& Geller, M. J. 1997, AJ, 113, 483

Saunders, W., Frenk, C., Rowan-Robinson, M., Lawrence, A., \& Efstathiou, G. 1991, Nature, 349, 32

Saunders, W., Sutherland, W. J., Maddox, S. J., et al. 2000, MNRAS, 317,55

Schechter, P. 1976, ApJ, 203, 297

Sheth, J. V., Sahni, V., Shandarin, S., \& Sathyaprakash, B. S. 2002, MNRAS, submitted [astro-ph/0210136]

Shepherd, C. W., Carlberg, R. G., Yee, H. K. C., et al. 2001, ApJ, 560, 72

Stoughton, C., Lupton, R. H., Bernardi, M., et al. 2002, AJ, 123, 485

Tucker, D. L., Oemler, A. Jr., Hashimoto, Y., et al. 2000, ApJS, 130, 237

Zeldovich, Ya. B., Einasto, J., \& Shandarin, S. F. 1982, Nature, 300, 407

Zwicky, F., Wield, P., Herzog, E., Karpowicz, M., \& Kowal, C. T. 1961-68, Catalogue of Galaxies and Clusters of Galaxies, 6 volumes. Pasadena, California Inst. Techn. 